\title{
CONTRIBUCIÓN AL ESTUDIO POLÍNICO DE ESPECIES ORNAMENTALES CON INTERÉS ALERGÓGENO CULTIVADAS EN MÁLAGA: DICOTILEDÓNEAS.
}

\author{
M. Mar TRIGO e Inmaculada FERNÁNDEZ
}

\begin{abstract}
RESUMEN. Contribución al estudio polínico de especies ornamentales con interés alergógeno cultivadas en Málaga: Dicotiledóneas. Se estudia con el microscopio óptico y con el microcopio electrónico de barrido la morfología polínica de 45 especies de plantas ornamentales habitualmente cultivadas en la ciudad de Málaga. Dichas especies presentan polinización de tipo anemófilo o anfífilo lo que las hace interesantes desde el punto de vista alergológico, habiendo sido citadas muchas de ellas como aerovagantes e incluso como productoras de polinosis por numerosos autores.
\end{abstract}

Palabras clave. Polen, ornamentales, dicotiledóneas, alergia.

ABSTRACT. Pollen morphology of ornamental species with aeroallergenic interest cultivated in Malaga (southern Spain). Due their allergenic potential, the pollen morphology of 45 species with anemophilous or anfiphilous pollination, cultivated in Malaga as ornamental plants, has been studied by light and scaning electon microscope. The pollen was acetolysed by the method of Erdtman (1960) modificated by Reitsma (1969) and mounted in glycerine jelly for light microcopy. For sacaning electron microscope the pollen were coated of evaporate gold .

Key words. Pollen, ornamental plants, dicotiledonees, allergy.

\section{INTRODUCCIÓN}

Cada día es mayor el número de personas que presenta sintomatología alérgica a algún tipo de polen, habiéndose incrementado considerablemente este número en Europa durante los últimos diez años, especialmente entre la población que habita en núcleos urbanos (D’Amato \& Spieksma, 1991). En estas áreas urbanas, son las plantas ornamentales junto con las malas hierbas las que integran la vegetación del entorno más próximo, de ahí el interés de su estudio, ya que son muchas las especies ornamentales cuyos pólenes han sido citados como aerovagantes, mientras que otras pertenecen a grupos de plantas tradicionalmente consideradas como alergógenas. Por otra parte, son las especies con polinización anemófila las que ofrecen mayor interés desde el punto de vista aerobiológico ya que emiten gran cantidad de polen con características aerodinámicas que favorecen su permanencia en la atmósfera.

Anteriormente ya se había abordado el estudio de la morfología polínica de las distintas especies de Gimnospermas cultivadas en 
Málaga (Trigo, 1989) por lo que, en el presente trabajo, se estudia la morfología polínica de 45 especies pertenecientes a 21 familias de dicotiledóneas con polinización en unos casos anemófila y en otros anfífila, como contribución al atlas polínico de su flora ornamental.

\section{MATERIAL Y MÉTODOS}

El estudio de los granos de polen se ha realizado utilizando tanto material fresco, incluido en ácido acético glacial, como material seco procedente del Herbario del Departamento de Biología Vegetal de la Universidad de Málaga (MGC). Dicho material se acetolizó siguiendo el método de Erdtman (1960) modificado por Reitsma (1969), si bien en algunos casos fue preciso rebajar la mezcla acetolítica a la proporción de 18:1 (anhidrido acético/ ácido sulfúrico) a causa de la debilidad que presentaba la exina de algunos pólenes.

Las preparaciones para el microscopio óptico (MO) se montaron en glicerogelatina, siguiendo el método de Wodehouse (1935), mientras que para su observación con el microscopio electrónico de barrido (MEB) las muestras se recubrieron con oro vaporizado en atmósfera de alto vacío, sin que en ningún caso fuera necesario utilizar un secador de punto crítico. En las microfotografías electrónicas aparecen, de izquierda a derecha, las siguientes inscripciones: número de archivo de la foto, kilovoltaje al que fue sometida la muestra, microescala y distancia de trabajo (WD) expresada en milímetros.

Para calcular las dimensiones de los ejes polar (P) y ecuatorial (E), así como el diámetro (D) en su caso, se han realizado 30 medidas y para el resto de los caracteres mesurables al menos 15. En los granos de polen polipantoporados, el número de poros se ha calculado por medio de la fórmula de Melville (1981). En la tabla 1 aparecen expresados los valores máximo y mínimo obtenidos de P, E, D y P/E y entre paréntesis la media aritmética \pm la desviación típica del intervalo.

La terminología empleada en la descripciones polínicas es básicamente la propuesta por Erdtman (1945, 1952), Faegri \& Iversen (1975), Hideux \& Ferguson (1975), Reitsma (1970) y Van Campo (1957, 1958), recogidas por Punt et al. (1994).

En el texto, las distintas especies aparecen dispuestas por familias, siguiendo éstas la ordenación filogenética de Stebbins (1974). En algunos casos se han agrupado varias especies cuando sus granos de polen presentaban una morfología similar. Para cada una de ellas se incluye su areal natural de distribución así como las referencias que tenemos sobre estudios anteriores tanto desde el punto de vista morfológico como alergógeno.

Material examinado: Acalypha willkesiana (MGC 16618, 17195). Acernegundo (MGC 16930, 17195). Ailanthus altissima (MGC 17374). Alternanthera bettzickiana (MGC 16936). Amaranthus caudatus (MGC 16899). Antigonon leptopus (MGC 17201). Atriplex halimus (MGC 16626). Broussonetia papyrifera (MGC 17012). Buxus balearica (MGC 17261). Buxus sempervirens (MGC 16986). Carya pekan (MGC 16611). Casuarina cuninghamiana (MGC 16680, 16666). Celosia argentea (MGC 17303). Celtis australis (MGC 17259). Ceratonia siliqua (MGC 16868). Cocculus laurifolius (MGC 17009). Fraxinus pensylvanica (MGC 17045, 17048). Gleditsia triacanthos (MGC 17335). Gomphrena globosa (17938). Juglans regia (MGC 16653). Kochia scoparia (MGC 17289). Ligustrum lucidum (MGC 17060, 17062). Ligustrum ovalifolium (MGC 17063). Morus alba (MGC 17028, 16586). Olea europaea (MGC 16552). Phytolacca dioica (MGC 17071). Pistacia lentiscus (MGC 17364). Platanus hispanica (MGC 17081, 17212). Populus alba (MGC 17208). Populus $x$ canadensis (MGC 17231). Quercus ilex (MGC 16615). Quercus robur (MGC 16616). Quercus rotundifolia (MGC 17310). Ricinus communis (MGC 17243). Salix x chrysocoma (MGC 17317). Salix x rubens (MGC 17214). Schinus molle (MGC 17211). Spondias mombin (MGC 16944). Tamarix 
africana (MGC 17158). Tamarix gallica (MGC 17157). Tamarix parviflora (MGC 17156, 16580). Tilia platyphyllos (MGC 17222). Tilia tomentosa (MGC 16898). Ulmus minor (MGC 17232). Ulmus glabra (MGC 17165).

\section{RESULTADOS}

\section{MENISPERMACEAE}

\section{Cocculus laurifolius DC. (Himalaya)}

Polen trizonocolporado. Isopolar, radiosimétrico. Circular en v.p. y c.o.e, ligeramente lobulado, sinuaperturado; elíptico en v. e. y c.o.m., de prolado a subprolado. Tamaño pequeño. Aberturas compuestas; ectoaberturas de tipo colpo, terminales, con la membrana apertural difícil de apreciar; endoaberturas de tipo poro, generalmente lolongadas, de 3-5 x 1,5-2 $\mu \mathrm{m}$, a veces circulares, de 1-2 $\mu \mathrm{m}$ de diámetro. Exina de 1-1,25 $\mu \mathrm{m}$ de grosor, con la sexina igual o ligeramente más gruesa que la nexina. Téctum parcial. Infratéctum columelado. Superficie uniformemente reticuladoperforada, con lúmenes más o menos circulares, siempre de menos de $1 \mu \mathrm{m}$ de diámetro y muros psilados (fig. 1: 1-3).

Referencias: Erdtman (1952), Harley \& Ferguson (1982), Pino y Díez(1990).

No se han encontrado referencias sobre la posible alergenicidad de esta especie. Tampoco ha sido citada como aerovagante.

\section{PLATANACEAE}

Platanus hispanica Miller ex Münchh. ( $P$. orientalis L. x P. occidentalis L.).

Polen trizonocolpado. Isopolar, radiosimétrico. De circular a ligeramente triangular en v.p. y c.o.e., planaperturado; de circular a ligeramente elíptico en v.e. y c.o.m., de subprolado a oblado-esferoidal. Tamaño pequeño. Aberturas simples, de tipo colpo, subterminales, de 8 a $10 \mu \mathrm{m}$ de longitud, con la membrana apertural granulosa. Exina de $1 \mu \mathrm{m}$ o menos de grosor, con la sexina ligeramente más gruesa que la nexina. Téctum parcial. Infratéctum columelado. Superficie reticulada, con lúmenes irregulares de hasta $1,5 \mu \mathrm{m}$ de longitud y los muros psilados (fig. 1: 4-6).

Referencias: Erdtman (1952), Domínguez et al. (1984), Pino y Díez (1990), La-Serna Ramos et al. (1991).
Especie citada como alergógena por Muñoz Medina (1949), Surinyach et al. (1955), Pla Dalmau (1961), Izco et al. (1972), Stanley \& Linskens (1974), Sáenz (1978), Gervais \& Millet (1978), Subiza (1980), Domínguez et al. (1984), Halse (1984) y Matthiesen et al. (1991). Citada además como aerovagante en Málaga por Cabezudo et al. (1994) y en numerosos calendarios polínicos (Spieksma, 1991) para el resto de Europa.

\section{FAGACEAE}

Quercus ilex L. (Región Mediterránea)

Quercus rotundifolia Lam. (Región Mediterránea)

Polen trizonocolporado (colporoidado). Isopolar, radiosimétrico. De circular a subtriangular en v.p. y c.o.e., angulaperturado; de circular a elíptico en v.e. y c.o.m. De prolado a obladoesferoidal. Tamaño de pequeño a mediano. Aberturas compuestas; ectoaberturas de tipo colpo, terminales, con la membrana apertural difícil de apreciar; endoaberturas de tipo poro, generalmente difusas. Exina de aproximadamente $2 \mu \mathrm{m}$ de grosor, con la sexina igual o ligeramente más gruesa que la nexina. Téctum contínuo. Infratéctum con columelas poco marcadas. Superficie aparentemente granulada al MO, apareciendo densamente cubierta por elementos irregulares de superficie rugulada cuando se observa con el MEB (fig.1: 7-10).

Referencias: Q. ilex: Pla Dalmau (1961), Sáenz (1973), Benthem et al. (1984), Saa et al. (1985). Q. rotundifolia: Sáenz (1973), Domínguez et al. (1984), Díez y Suárez (1987).

Quercus robur L. (Europa y Asia occidental)

Polen trizonocolporado (colporoidado). Isopolar, radiosimétrico. De circular a ligeramente lobulado o subtriangular en v.p. y c.o.e., angulaperturado; de circular a elíptico en v.e. y c.o.m. De prolado a prolado-esferoidal. Tamaño de pequeño a mediano. Aberturas compuestas; ectoaberturas de tipo colpo, terminales, con la membrana ornamentada con elementos irregulares; endoaberturas de contorno difuso. Exina de 1,5-2 $\mu \mathrm{m}$ de grosor, con la sexina aproximadamente $\tan$ gruesa como la nexina. Téctum contínuo. Infratéctum columelado, con columelas poco marcadas. Superficie granulosa (fig. 1:11-14).

Referencias: Pla Dalmau (1961), Benthem et al. (1984), Saa et al. (1985). 
Las especies del género Quercus han sido citadas como alergógenas por Muñoz Medina (1949), Pla Dalmau (1961), Izco et al. (1972), Sáenz (1978), Subiza (1980), Domínguez et al. (1984), Lewis et al. (1984), Halse (1984) y Matthiesen et al. (1991). También se encuentran citadas como aerovagantes en Málaga por Cabezudo et al. (1994) y en otros trabajos de aerobiología de toda Europa (Spieksma, 1991; Ickovic \& Thibaudon, 1991).

\section{CASUARINACEAE}

Casuarina cuninghamiana Miq. (Australia).

Polen trizonoporado. Isopolar, radiosimétrico. Triangular en v.p. y c.o.e., angulaperturado; elíptico en v.e. y c.o.m., suboblado. Tamaño mediano. Aberturas simples, de tipo poro, circulares, de 2 a $2,5 \mu \mathrm{m}$ de diámetro, situadas en la zona ecuatorial, aspidadas. Exina de 1,5-2 $\mu \mathrm{m}$ de grosor, con la sexina dos veces más gruesa que la nexina. Téctum contínuo. Infratéctum columelado. Superficie equinulada, apareciendo a veces pequeñas rúgulas, en general poco marcadas, cuando se observan los granos de polen con el MEB (fig.1: 15-17).

Referencias: Erdtman (1952), Lewis et al. (1983), La-Serna Ramos et al. (1991).

Especie citada como alergógena por Stanley \& Linskens (1974) y Lewis et al. (1983). Su capacidad alergizante ha sido recientemente puesta de manifiesto por Bucholtz et al. (1987). Citada como aerovagante en Málaga por Cabezudo et al. (1994).

\section{AMARANTHACEAE}

Alternanthera bettzickiana Nichols (Brasil).

Polen polipantoporado. Apolar, radiosimétrico. De circular a poligonal en corte óptico, esferoidal. Tamaño pequeño. Aberturas simples de tipo poro, generalmente en número de 12 por grano de polen, de $5-7,5 \mu \mathrm{m}$ de diámetro y contorno ligeramente poligonal, con la membrana apertural granulosa. Exina de 1,5-2 $\mu \mathrm{m}$ de grosor, con la sexina mucho más gruesa que la nexina. Téctum contínuo. Infratéctum columelado. Superficie psilado-perforada, apareciendo espínulas como elementos supratectales (fig. 2: 1-3).

Referencias: se desconocen.

No se han encontrado referencias sobre la posible alergenicidad de esta especie. Tampoco ha sido citada como aerovagante, a pesar de que su morfología polínica impide la confusión con el tipo
Chenopodiaceae-Amaranthaceae habitualmente citado por los aerobiólogos.

\section{Gomphrena globosa L. (América tropical)}

Polen polipantoporado. Apolar, radiosimétrico. Circular en corte óptico, esferoidal. Tamaño de pequeño a mediano. Aberturas simples, de tipo poro y contorno más o menos circular, de aproximadamente $2 \mu \mathrm{m}$ de diámetro, dispuestas en número de 50-65 por grano de polen. Membrana apertural granuloso-equinulada. Exina de 4-4,5 $\mu \mathrm{m}$ de grosor, con la sexina 3 veces más gruesa que la nexina. Téctum parcial, muy grueso $(2-2,5 \mu \mathrm{m})$. Infratéctum columelado, con columelas cortas. Superficie reticulada, con lúmenes exagonales de aproximadamente $4 \mu \mathrm{m}$ de diámetro y muros psilados, pudiéndose apreciar a través de los lúmenes las columelas del infratéctum (fig. 2: 4-5).

Referencias: Se desconocen.

No se han encontrado referencias sobre la posible alergenicidad de esta especie. Tampoco ha sido citada como aerovagante. Su particular morfología polínica impide la confusión con el tipo Chenopodiaceae-Amaranthaceae habitualmente citado por los aerobiólogos.

\section{Amaranthus caudatus L. (Subcosmopolita)}

Polen polipantoporado. Apolar, radiosimétrico. De circular a ligeramente elíptico en corte óptico. Esferoidal. Tamaño de pequeño a mediano. Aberturas simples, de tipo poro, de 1-1,5 $\mu$ m de diámetro, dispuestas en número de 32 - 45 por grano de polen y regularmente distribuidas por toda la superficie. Membrana apertural granulosa. Exina de 1,5 $\mu \mathrm{m}$ de grosor, con la sexina aproximadamente tan gruesa como la nexina. Téctum contínuo. Infratéctum columelado. Superficie densamente equinulada (fig. 2: 6-8)

Referencias: Se desconocen.

El género Amaranthus ha sido citado como alergógeno por Sáenz (1978), Gervais \& Millet (1978) y Matthiesen et al. (1991).

\section{Celosia argentea L. (Asia)}

Polen polipantoporado. Apolar, radiosimétrico. Circular en corte óptico. Esferoidal. Tamaño de pequeño a mediano. Aberturas simples de tipo poro, circulares de 3-4 $\mu \mathrm{m}$ de diámetro, dispuestas en número de 25-70 por grano de polen. Membrana apertural equinulada, que a menudo se pierde con la 


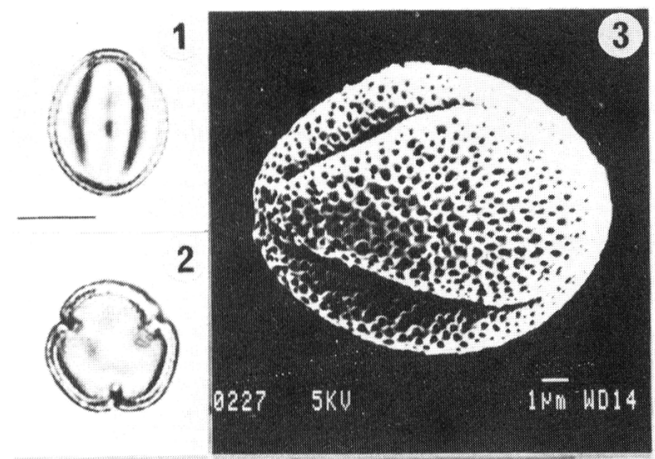

(3)
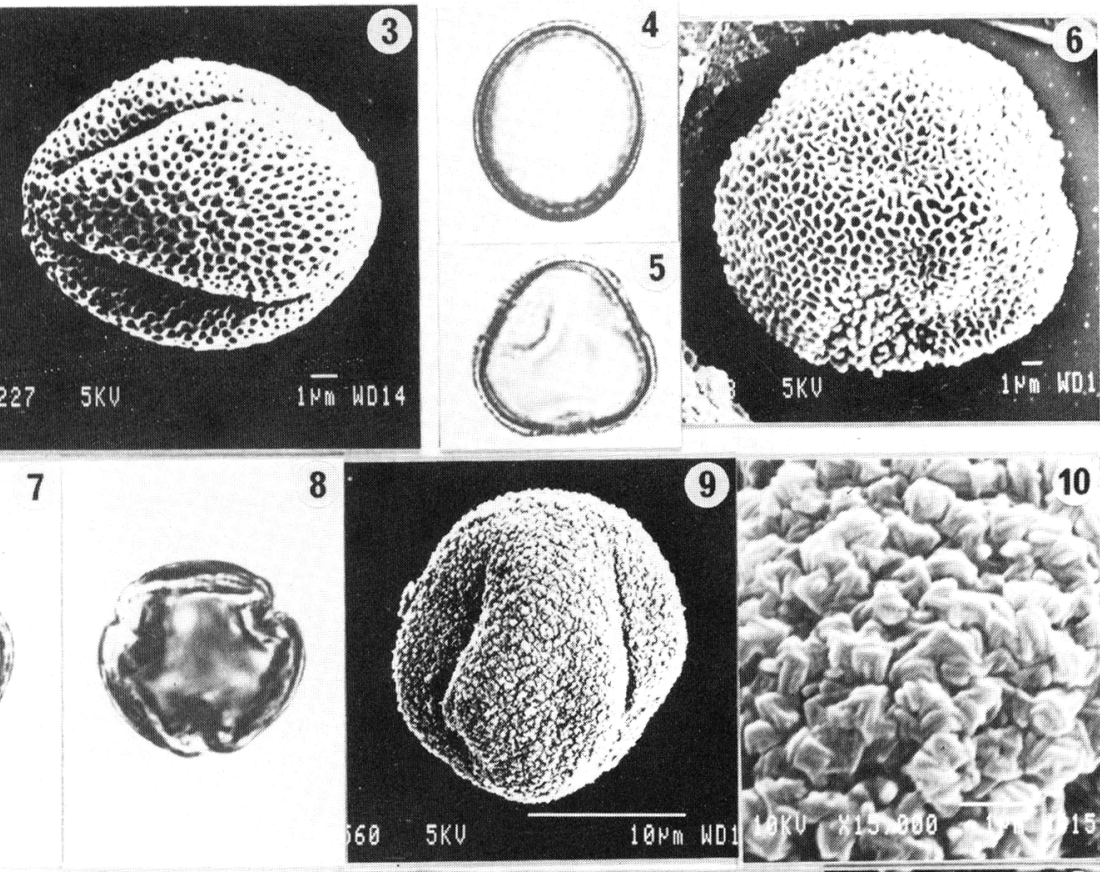

11
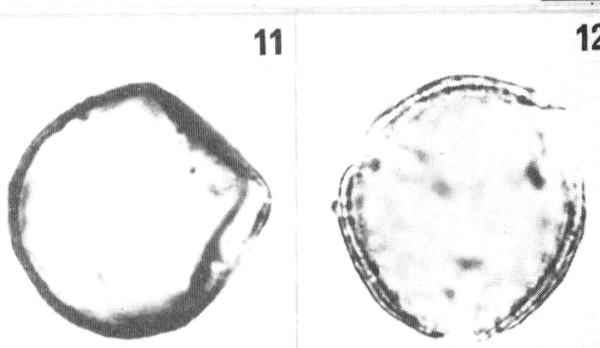

12

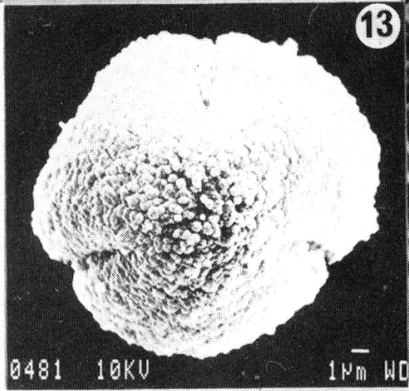

(13) $0.27,14$

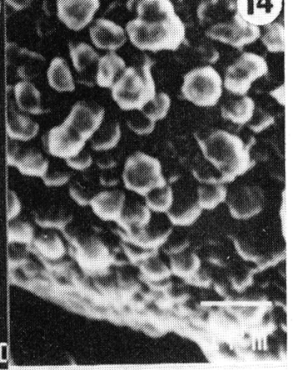

15
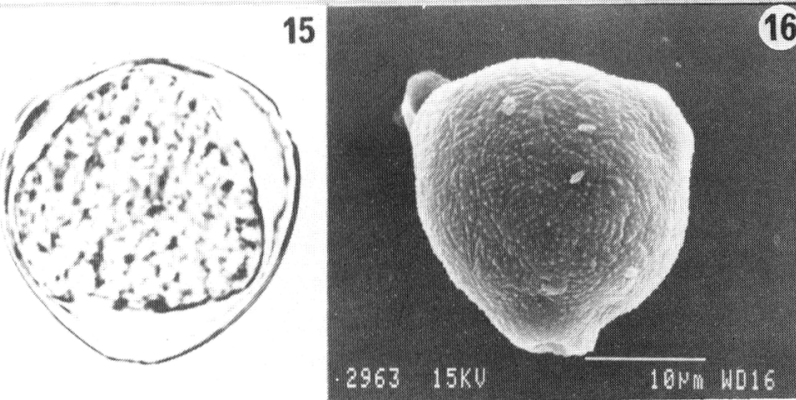

16

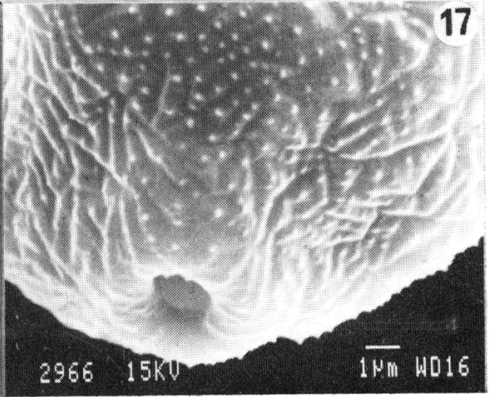

Figura 1. Cocculus laurifolius:1) v.e. y c.o.m. 2) v.p. y c.o.e. 3) Aspecto general del polen. Platanus hispanica: 4) v.e. y c.o.m. 5) v.p. y c.o.e. 6) Aspecto general del polen. Quercus ilex: 7) v.e. y c.o.m. 8) v.p. y c.o.e. 9) Aspecto general del polen. 10) Detalle de la ornamentación. Quercus robur:11) v.e. y c.o.m. 12) v.p. y c.o.e.; 13) Aspecto general del polen. 14) Detalle de la ornamentación. Casuarina cuninghamiana:

15) v.p. y c.o.e. 16) Aspecto general del polen. 17) Detalle de la ornamentación y abertura. Escala MO: $10 \mu \mathrm{m}$. 
acetolisis. Exina de 2,5-3 $\mu \mathrm{m}$ de grosor, con la sexina de 4-5 veces más gruesa que la nexina. Téctum contínuo. Infratéctum columelado. Superficie psilado-perforada. Como elementos supratectales aparecen espínulas de muy pequeño tamaño (fig. 2: 9-10).

Referencias: Se desconocen.

Las especie del género Celosia han sido citadas como altamente alergógenas por Lewis et al. (1983).

La familia Amaranthaceae en conjunto ha cido citada como alergógena por Lewis et al. (1983), Domínguez et al. (1984) y King \& Norman (1986). Al tratarse de una familia cosmopolita, sus especies, junto con las de Chenopodiaceae, se incluyen como aerovagantes en la mayoría de los trabajos sobre aerobiología (Spieksma, 1991).

\section{PHYTOLACCACEAE}

\section{Phytolacca dioica L. (Sudamérica)}

Polen trizonocolpado. Isopolar, radiosimétrico. De subcircular a ligeramente lobulado en v.p. y c.o.e., sinuaperturado; elíptico en v.e. y c.o.m. De prolado a subprolado. Tamaño mediano. Aberturas simples de tipo colpo, terminales. Membrana apertural granulosa. Exina de c. 1,5 $\mu \mathrm{m}$ de grosor, con la sexina mucho más gruesa que la nexina. Téctum contínuo. Infratéctum columelado. Superficie perforada, apareciendo espínulas como elementos supratectales (fig. 2: 11-14).

Referencias: Pino y Díez (1990).

No se han encontrado referencias sobre la posible alergenicidad de esta especie. Tampoco ha sido citada como aerovagante.

\section{CHENOPODIACEAE}

Atriplex halimus L. (Región Mediterránea)

Kochia scoparia Schrad. (Europa y Asia)

Polen polipantoporado. Apolar, radiosimétrico. De circular a ligeramente elíptico en corte óptico. Generalmente esferoidal. Tamaño de pequeño a mediano. Aberturas simples, de tipo poro, circulares, de $1,5 \mu \mathrm{m}$ de diámetro en $A$. halimus y de $2-2,5$ $\mu \mathrm{m}$ en $K$. scoparia, dispuestos en $\mathrm{n}^{\circ}$ de $100-120$ por grano de polen y regularmente distribuidos por toda la superficie. Membrana apertural granulosa en $A$. halimus y equinulada en $K$. scoparia. Exina de 2-2,5 $\mu \mathrm{m}$ de grosor, con la sexina más gruesa que la nexina. Téctum contínuo. Infratéctum columelado. Superficie equinulada (fig. 2: 15-17) Referencias: A. halimus: Candau (1987a). K. scoparia: Erdtman (1952), Lewis et al. (1983).

La familia Chenopodiaceae en conjunto ha sido citada como alergógena por numerosos autores, apareciendo junto con Amaranthaceae en la mayoría de los trabajos sobre muestreos aerobiológicos (Spieksma, 1991). Los géneros Kochia y Atriplex son considerados como tal por Durhan (1945), Lewis et al. (1983), King \& Norman (1986) y Matthiesen et al. (1991).

\section{POLYGONACEAE}

\section{Antigonon leptopus Hook. et Arm. (México)}

Polen trizonocolporado. Isopolar, radiosimétrico. Circular lobulado en v.p. y c.o.e; elíptico en v.e. y c.o.m. De prolado a prolado-esferoidal. Tamaño pequeño. Aberturas compuestas; ectoaberturas de tipo colpo, terminales; endoaberturas de tipo poro, lalongadas, lineares de 1-1,5 x 4-7 $\mu \mathrm{m}$. Exina de 2$2,5 \mu \mathrm{m}$ de grosor, con la sexina tan gruesa como la nexina, formando ésta última costillas a nivel de las endoaberturas. Téctum contínuo. Infratéctum columelado. Superficie equinulada (fig. 3: 1-5).

Referencias: se desconocen.

No se han encontrado referencias sobre la posible alergenicidad de esta especie en concreto, aunque muchas poligonáceas son citadas habitualmente como alergógenas y aerovagantes.

\section{TILIACEAE}

Tilia platyphyllos Scop. (Centro y Sur de Europa) Tilia tomentosa Moench. (Este de Europa y Asia Menor)

Polen trizonocolporado. Isopolar, radiosimétrico. De subircular a triangular en v.p. y c.o.e, planaperturado; elíptico en v.e. y c.o.m. De peroblado a oblado. Tamaño de mediano a grande. Aberturas compuestas; ectoaberturas de tipo colpo, muy cortos y situados en la zona ecuatorial, de 6-8 $\mu \mathrm{m}$ de longitud; endoaberturas de tipo poro, circulares, de 2-3 $\mu \mathrm{m}$ de diámetro, apareciendo un pequeño vestíbulo. Exina de aproximadamente $3 \mu \mathrm{m}$ de grosor, con la sexina ligeramente más gruesa que la nexina, engrosándose ésta última a nivel de las endoaberturas para formar costillas muy gruesas. Téctum parcial. Infratéctum columelado. Superficie perforado-reticulada, con lúmenes de hasta $1 \mu \mathrm{m}$ de diámetro. Como elementos supratectales pueden aparecer pequeños gránulos (fig. 3: 6-9). 

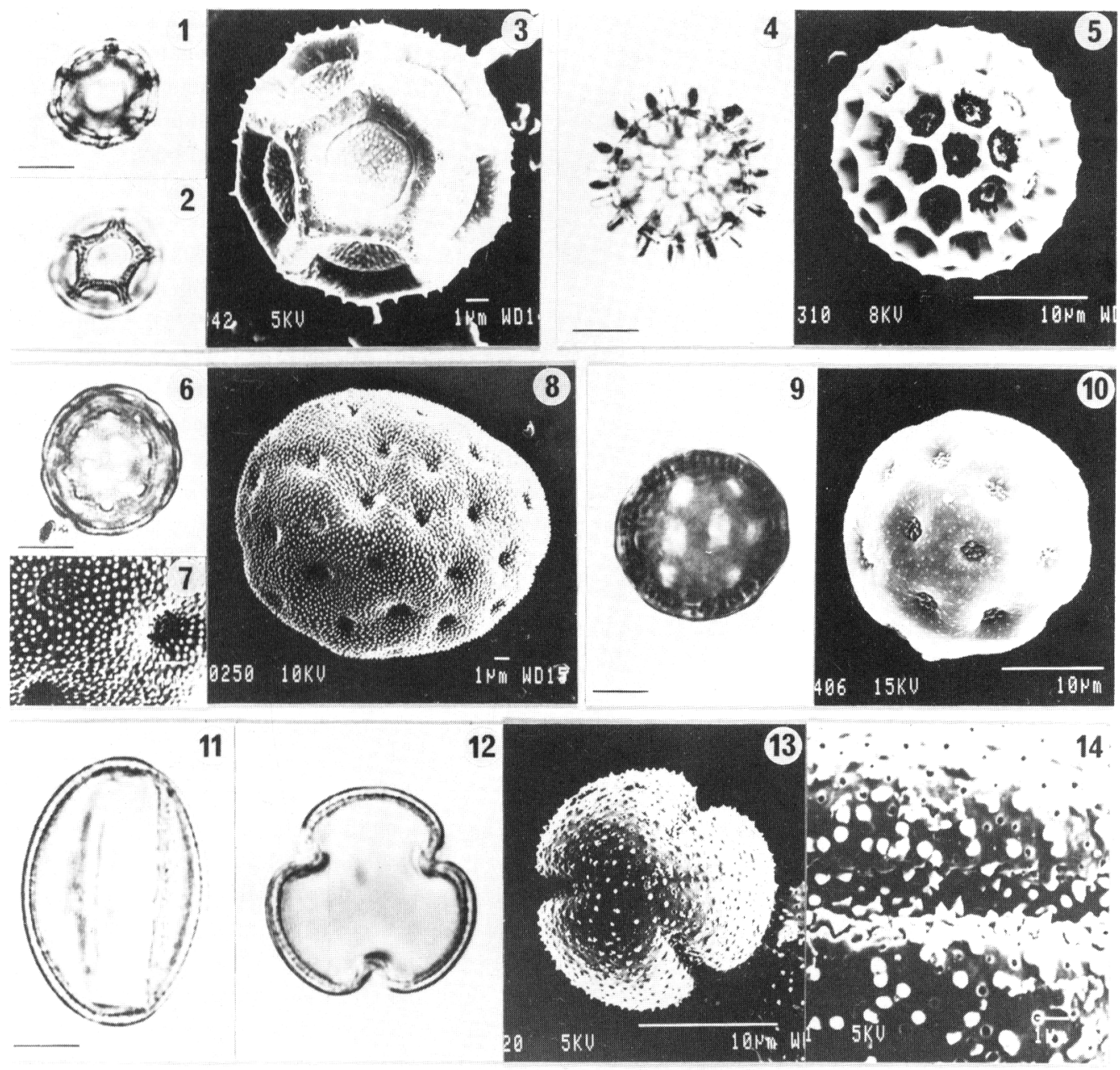

(13)
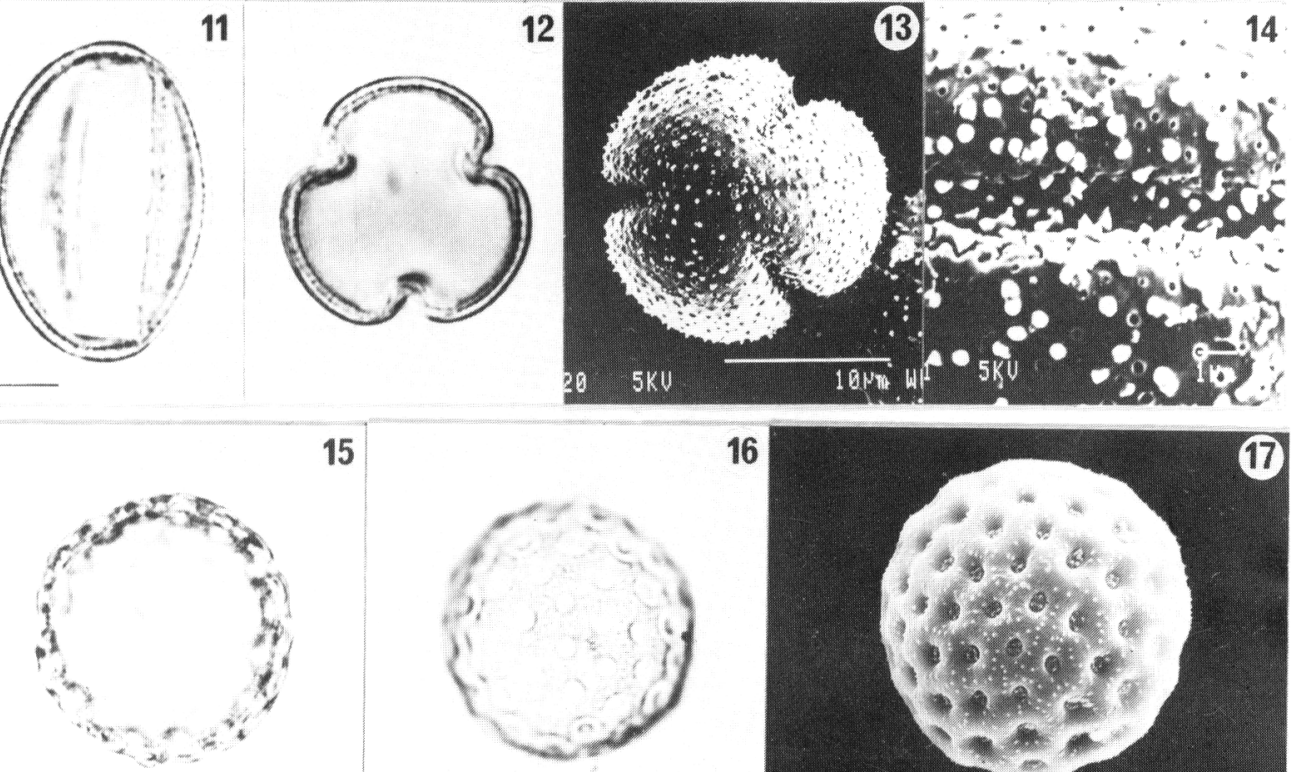

15

16

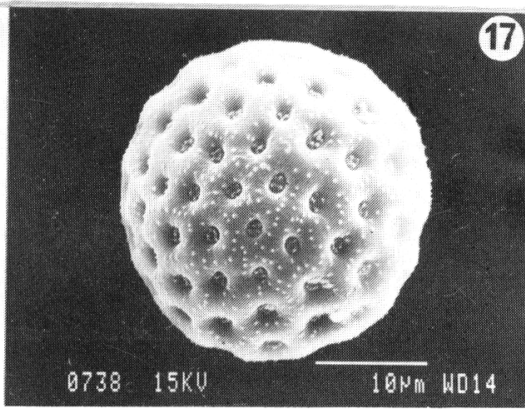

Figura 2. Alternanthera bettzickiana: 1) c.o. 2) Superficie y abertura. 3) Aspecto general del polen. Gomphrena globosa: 4) c.o. 5) aspecto general del polen. Amaranthus caudatus: 6) c.o. 7) Detalle de la superficie. 8) Aspecto general del polen. Celosia argentea: 9) c.o. 10) Aspecto general del polen. Phytolacca dioica: 11) v.e. y c.o.m. 12) v.p. y c.o.e. 13) Aspecto general del polen. 14) Detalle de la superficie y abertura. Kochia scoparia: 15) c.0. 16) Superficie. 17) Aspecto general del polen. Escala MO: $10 \mu \mathrm{m}$. 
Referencias: $T$. platyphyllos: Pla Dalmau (1961), Gálvez y Ubera (1985), Christensen \& Blackmore (1988), Pino y Díez (1990), Bueno et al. (1993). T. tomentosa: Erdtman (1952), La-Serna Ramos et al. (1991).

Especies del género Tilia han sido citadas como alergógenas por Muñoz Medina (1949), Pla Dalmau (1961), Sáenz (1978) y Lewis et al. (1983) entre otros. Igualmente son numerosos los trabajos sobre aerobiaología en que sus pólenes se citan como aerovagantes.

\section{ULMACEAE}

\section{Celtis australis L. (Región Mediterránea)}

Polen generalmente tetrazonoporado, a veces trizonoporado o polipantoporado. Isopolar o apolar, radiosimétrico. Subcircular en corte óptico. Esferoidal. Tamaño mediano. Aberturas simples de tipo poro, generalmente en $n^{\circ}$ de 4 y situadas en la zona ecuatorial por pares opuestos, a veces 3 ó más de 4, de 1-2 $\mu \mathrm{m}$ de diámetro. Exina de 2-3 $\mu \mathrm{m}$ de grosor, formando un anillo alrededor de los poros, con la sexina tan gruesa como la nexina. Téctum contínuo. Infratéctum columelado. Superficie escábrida, con pequeñas perforaciones. Aparecen espínulas, de muy pequeño tamaño como elementos supratectales (fig. 3: 10-12).

Referencias: Díez (1987a).

Especie citada como alergógena por Sáenz (1978), Lewis et al. (1983) y Rogers (1989); y como aerovagante por Belmonte i Soler (1988) y O'Rourke (1989)

\section{Ulmus glabra Hudson (Europa)}

Ulmus minor Miller (Europa, norte de África y oeste de Asia)

Polen de penta- a heptazonoporado, siendo más frecuente el tipo hexazonoporado en $U$. glabra y el pentazonoporado en $U$. minor. Isopolar, radiosimétrico. De subcircular a poligonal (pentagonal a heptagonal) en v.p. y c.o.e., angulaperturado; elíptico en v.e. y c.o.m. De suboblado a oblado. Tamaño mediano. Aberturas simples, de tipo poro, de aproximadamente $1 \mu \mathrm{m}$ de diámetro, situadas en la zona ecuatorial y rodeadas por un anillo. Membrana apertural granulosa. Exina de c. $1,5 \mu \mathrm{m}$ de grosor en la mesoporía y de aproxi- madamente 2,5 $\mu \mathrm{m}$ alrededor de los poros, con sexina y nexina difíciles de distinguir con el M.O. Téctum contínuo. Infratéctum columelado. Superficie rugulada. Como elementos supratectales aparecen pequeños gránulos (fig. 3: 13-15).

Referencias: U. glabra: Stockmarr (1974), Zavada (1983). U. minor: Stockmarr (1974), Domínguez et al. (1984), Díez (1987a).

Especies citadas como alergógenas por Muñoz Medina (1949), Izco et al. (1972), Stanley \& Linskens (1974), Michel et al. (1978), Sáenz (1978), Lewis et al. (1983), Domínguez et al. (1984) y Matthiesen et al. (1991). Las especies del género Ulmus han sido ampliamente citadas como aerovagantes en gran número de trabajos sobre aerobiología (Spieksma, 1991). Citado para Málaga por Cabezudo et al. (1994).

\section{MORACEAE}

Broussonetia papyrifera (L.) Vent. (Japón)

Morus alba L. (Asia oriental)

Polen di-(tri-)zonoporado. Isopolar, radiosimétrico o bisimétrico. Circular tanto en v.p. y c.o.e. como en v.e. y c.o.m. Esferoidal. Tamaño pequeño. Aberturas simples, de tipo poro, de 2-3,5 $\mu \mathrm{m}$ de diámetro en $B$. papyrifera y de 1,5-2 $\mu \mathrm{m}$ de diámetro en M. alba, provistos de opérculo que se pierde fácilmente con la acetolisis. Exina muy delgada, de menos de $1 \mu \mathrm{m}$ de grosor, con la sexina tan gruesa como la nexina. Téctum contínuo. Infratéctum con columelas poco aparentes. Superficie equinulada (fig. 4: 1-6).

Referencias: B. papyrifera: Lieux (1980), Lewis et al. (1983), Domínguez et al. (1984), Gálvez y Ubera (1985), Pino y Díez (1990). M. alba: Pla Dalmau (1961), Lewis et al. (I.c.), Punt \& Malatoux (1984), Domínguez et al. (1984), Candau (1987b).

B. papyrifera ha sido citada como alergógena por Stanley \& Linskens (1974), Sáenz (1978), Lewis et al. (1983) y Domínguez et al. (1984). Y M. alba por Pla Dalmau (1961), Stanley \& Linskens (1974), Sáenz (1978), Al-Doory et al. (1980), Singh \& Babu (1980), Lewis et al. (1983), Halse (1984), Domínguez et al. (1984) y Matthiesen et al. (1991). Tanto una como otra especie son ampliamente citadas como aerovagantes en numerosos estudios aerobiológicos. El tipo Morus ha sido citado para 

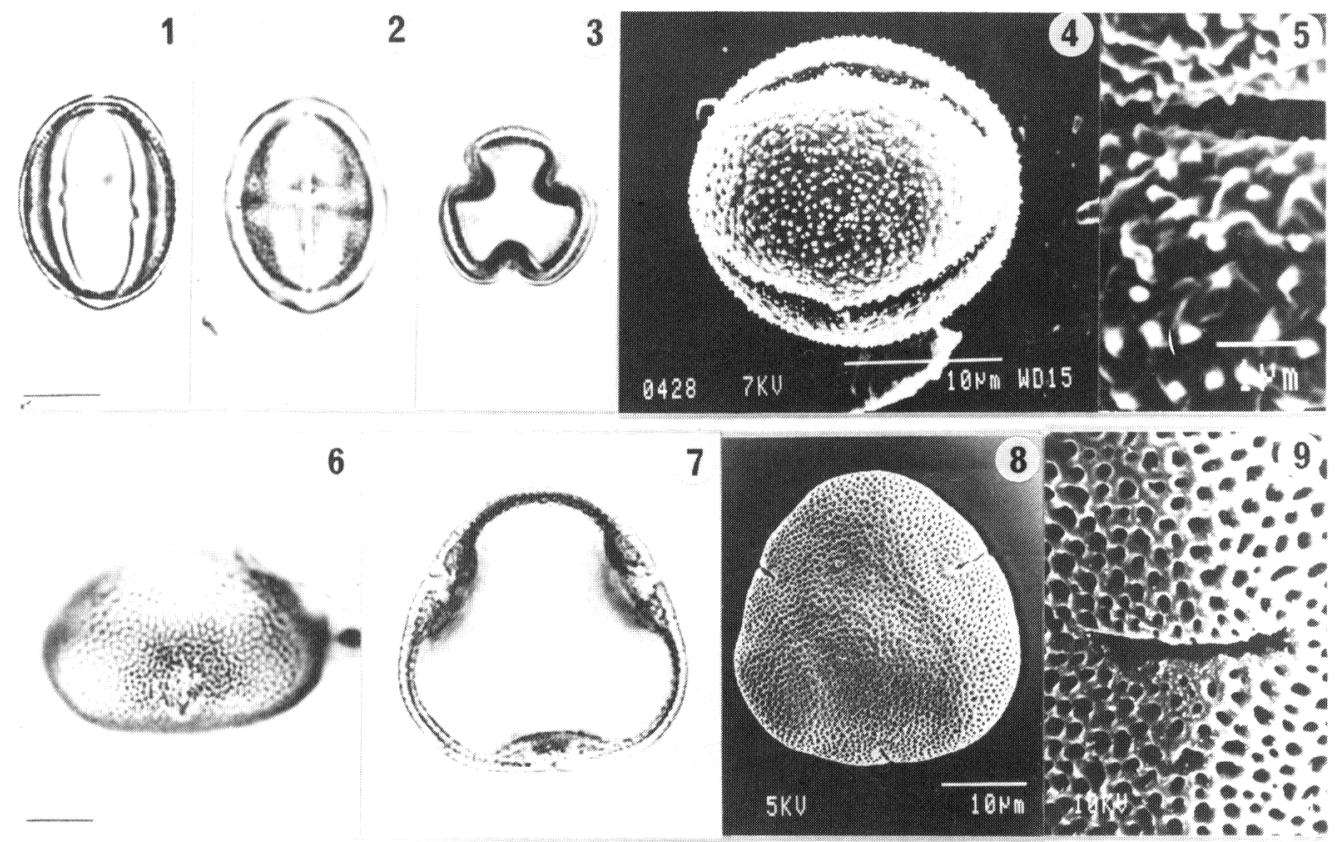

10

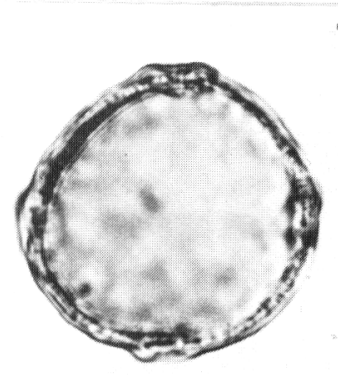

11
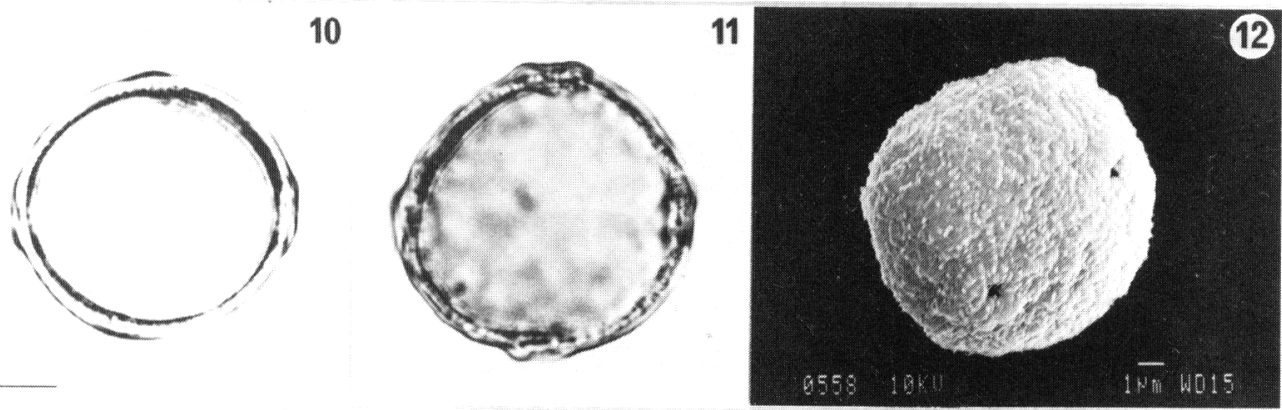

13
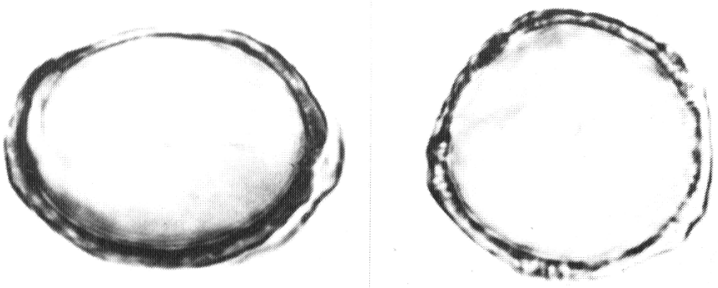

14

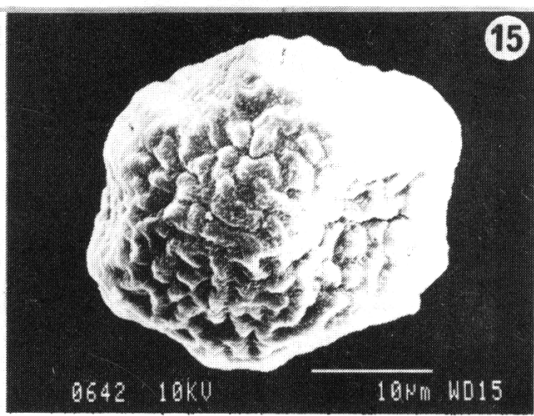

Figura 3. Antigonon leptopus: 1) v.e. y c.o.m. 2) Abertura 3) v.p. y c.o.e. 4) Aspecto general del polen. 5) Detalle de la superficie. Tilia tomentosa: 6) superficie y abertura. 7) v.p. y c.o.e. 8) Aspecto general del polen 9) Detalle de la abertura y ornamentación. Celtis australis: 10) v.e. y c.o.m. 11) v.p. y c.o.e. 12) Aspecto general del polen. Ulmus minor: 13) v.e. y c.o.m. Ulmus glabra: 14) v.p. y c.o.e. 15) Aspecto general del polen. Escala MO: $10 \mu \mathrm{m}$. 
Málaga por Cabezudo et al. (1994).

\section{TAMARICACEAE}

Tamarix africana Poir. (Región Mediterránea)

Tamarix gallica L. (sur de Europa)

Tamarix parviflora DC. (Región Egea)

Polen trizonocolporado. Isopolar, radiosimétrico. Circular lobulado en v.p. y c.o.e., sinuaperturado; elíptico obtuso en v.e. y c.o.m. De prolado a obladoesferoidal. Tamaño pequeño. Aberturas simples, de tipo colpo, terminales. Exina de aproximadamente $1,5 \mu \mathrm{m}$ de grosor, con la sexina tan gruesa como la nexina. Téctum parcial. Infratéctum columelado, con columelas gruesas. Superficie reticulada, con lúmenes irregulares de hasta $1 \mu \mathrm{m}$ de longitud y los muros psilados (fig. 4: 7-10).

Referencias: T. africana: Sáenz (1980), Fernández (1987a). T. gallica: Erdtman (1952), Pla Dalmau (1961), Fernández (l.c.). T. parviflora: se desconocen.

El género Tamarix en su conjunto ha sido considerado como alergógeno por Lewis et al. (1983). Halse (1984) cita como especie causante de polinosis a $T$. gallica.

\section{SALICACEAE}

Polpulus alba L. (Europa, Asia y norte de África) Polpulus x canadensis Moench. (P. nigra L. x $P$. deltoides Marshall)

Polen inaperturado. Apolar, radiosimétrico. Circular en corte óptico. Esferoidal. Tamaño de pequeño a mediano. Exina de aproximadamente 1 $\mu \mathrm{m}$ de grosor, con la sexina más gruesa que la nexina. Téctum parcial. Infratéctum con columelas poco patentes. Superficie finamente perforadoreticulada, con lúmenes de menos de $1 \mu \mathrm{m}$ de diámetro y muros irregulares. Aparecen espínulas, de muy pequeño tamaño, como elementos supratectales (fig. 4: 11-13).

Referencias: P. alba: Pla Dalmau (1961), Díez (1987b). P. x canadensis: se desconocen.

Las especies del género Populus han sido ampliamente citadas como alergógenas por numerosos autores: Surinyach et al. (1955), Pla Dalmau (1961), Stanley \& Linskens (1974), Sáenz (1978), Lewis et al. (1983), Domínguez et al. (1984), Halse (1984) y Matthiesen et al. (1991). Igualmente sus pólenes son habitualmente citados como aerovagantes (Spieksma, 1991). El tipo Populus ha sido citado para Málaga por Cabezudo et al. (1994).
Salix x chrysocoma Dode (S. alba L. subsp. vitellina (L.) Arcangeli $x$ S. babylonica L.)

Salix x rubens Schrank (S. alba L. x S. fragilis L.)

Polen trizonocolporado (colporoidado). Isopolar, radiosimétrico. Circular lobulado en v.p. y c.o.e., sinuaperturado. Elíptico en v.e. y c.o.m., rara vez circular. De prolado a prolado-esferoidal. Tamaño de pequeño a mediano. Aberturas compuestas; ectoaberturas de tipo colpo, terminales, apareciendo a veces endoaberturas difusas y poco apreciables en la zona central del colpo, lolongadas. Membrana apertural formada por elementos poliédricos e irregulares. Exina de 1-1,5 $\mu \mathrm{m}$ de grosor, con la sexina 2 veces más gruesa que la nexina. Téctum parcial. Infratéctum columelado, con columelas gruesas. Superficie ampliamente reticulada, con lúmenes muy irregulares de hasta 2,5 $\mu \mathrm{m}$ de diámetro en la mesocolpía, decreciendo en tamaño hacia los colpos y zonas polares y estando ornamentados por la presencia de gemas; muros crestados. A través de los lúmenes se aprecian las columelas del infratéctum (fig. 4: 14-17).

Referencias: Se desconocen.

El género Salix ha sido citado como alergógeno por Surinyach et al. (1955); Stanley \& Linskens (1974), Lewis et al. (1983), Domínguez et al. (1984), Halse (1984) y Matthiesen et al. (1991). El polen de estas especies ha sido ampliamente citado como aerovagante (Spieksma, 1991).

\section{FABACEAE}

\section{Ceratonia siliqua L. (Región Mediterránea)}

Polen tetrazonocolporado, a veces trizonocolporado y rara vez pentazonocolporado. Isopolar, a veces heteropolar, radiosimétrico. Subcircular en v.p. y c.o.e., de elíptico a subcircular en v.e. y c.o.m. De prolado a suboblado. Tamaño de pequeño a mediano. Aberturas compuestas; ectoaberturas de tipo colpo terminales; endoaberturas de tipo poro, lalongadas, elípticas de 5-7 x 10-12 $\mu \mathrm{m}$. Membrana apertural granulosa. A veces los colpos se fusionan 2 a 2 en uno de los polos (loxocolporado), en tales casos los granos de polen serían heteropolares. Exina de aproximadamente $2,5 \mu \mathrm{m}$ de grosor, con la sexina ligeramente más gruesa que la nexina, formando ésta última costillas a nivel de las endoaberturas. Téctum contínuo. Infratéctum columelado. Superficie perforada (fig. 5: 1-4).

Referencias: Pla Dalmau (1961), Ferguson (1980), Fernández (1987b). Especie citada como 

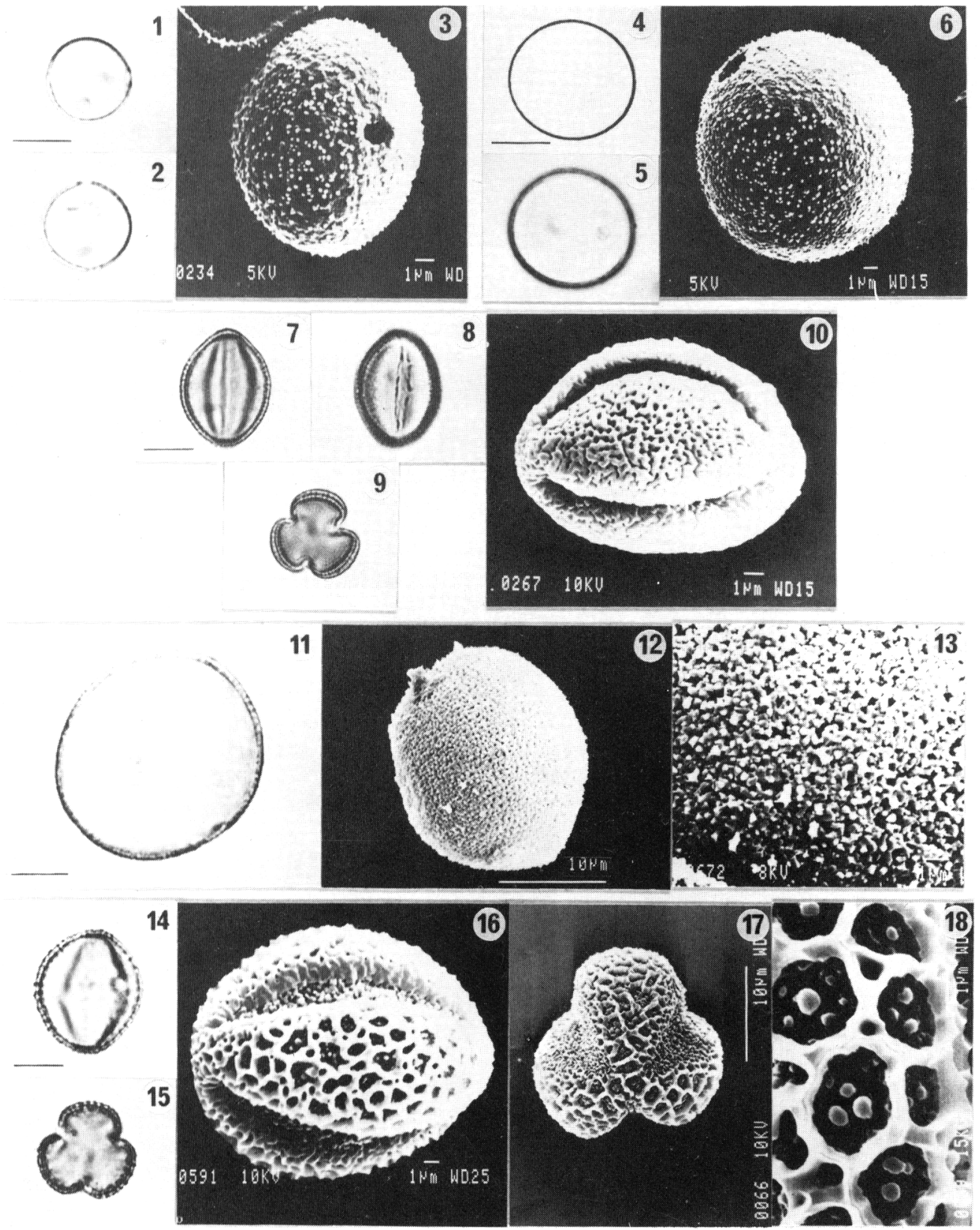

Figura 4. Broussonetia papyrifera: 1) v.e. y c.o.m. 2) v.p. y c.o.e. 3) Aspecto general del polen. Morus alba: 4) v.e. y c.o.m. 5) Abertura. 6) Aspecto general del polen. Tamarix gallica: 7) v.e. y c.o.m. 9) v.p. y c.o.e. Tamarix africana: 8) Abertura. 10) Aspecto general del polen. Populus $x$ canadensis: 11) c.o. 12) Aspecto general del polen. 13) Detalle de la superficie. Salix x chrysocoma: 14) v.e. y c.o.m. 15) v.p. y c.o.e. 16) Aspecto general del polen. Salix $x$ rubens: 17) Aspecto general del polen. 18) Detalle de la ornamentación. Escala MO: $10 \mu \mathrm{m}$. 
alergógena por Halse (1984) y como aerovagante por Torreguitart Zorrilla et al. (1990).

Gleditsia triacanthos L. (Sur de Estados Unidos) Polen trizonocolporado. Isopolar, radiosimétrico. Circular lobulado en v.p. y c.o.e., sinuaperturado; elíptico en v.e. y c.o.m. De prolado a proladoesferoidal. Tamaño mediano. Aberturas compuestas; ectoaberturas de tipo colpo, terminales, bordeadas de un estrecho margo psilado-perforado; endoaberturas bastante difusas y más o menos circulares. Membrana apertural escábrida. Exina de 1$1,5 \mu \mathrm{m}$ de grosor, con la sexina de 1,5 a 2 veces más gruesa que la nexina. Téctum parcial. Infratéctum columelado. Superficie reticulada, con lúmenes irregulares de hasta $2 \mu \mathrm{m}$ de longitud, disminuyendo el tamaño de los mismos a mediada que se aproximasn a los bordes de los colpos, donde la superficie se hace perforada; muros muy estrechos, psilados (fig. 5: 5-7).

Referencias: Ferguson (1987), Pardo (1990).

Especie citada como alergógena por Sáenz (1978), Lewis et al. (1983) y Halse (1984).

\section{BUXACEAE}

Buxus balearica Willd. (Cerdeña, Baleares y sur de la Península Ibérica)

Buxus sempervirens L. (Europa, norte de África y oeste de Asia)

Polen polipantoporado. Apolar, radiosimétrico. Circular en corte óptico. Esferoidal. Tamaño mediano. Aberturas simples de tipo poro, en número de 25-65 por grano de polen, de contorno irregular, pero más o menos circulares, a menudo difusos. Membrana apertural granulosa. Exina de 1-2 $\mu \mathrm{m}$ de grosor, con la sexina 1,5 veces tan gruesa como la nexina. Téctum parcial. Infratéctum columelado. Superficie perforado-reticulada, con lúmenes irregulares y anastomosados, de menos de $1 \mu \mathrm{m}$ de longitud y muros equinulados (fig. 5: 8-10).

Referencias: B. balearica Erdtman (1952), Mateu et al. (1987), Trigo et al. (1992). B. sempervirens: Erdtman (1952), Lewis et al. (1983).

El género Buxus es citado como alergógeno por Lewis et al. (1983) y como aerovagante por Belmonte i Soler (1988) y Torreguitart Zorrilla et al. (1990).

\section{EUPHORBIACEAE}

Acalypha willkesiana Muell. (América tropical)
Polen trizonocolporado. Isopolar, radiosimétrico. Circular lobulado en v.p. y c.o.e., sinuaperturado; elíptico obtuso en v.e. y c.o.m. De prolado a obladoesferoidal. Tamaño de pequeño a mediano. Aberturas compuestas; ectoaberturas de tipo colpo, terminales, constreñidas en la zona ecuatorial a nivel de las endoaberturas; endoaberturas de tipo poro, muy reducidas y circulares. Exina de c. 1,5 $\mu \mathrm{m}$ de grosor, con la sexina 1,5 veces tan gruesa como la nexina. formando ésta ultima costillas a nivel de las endoaberturas. Aparece también un pequeño vestíbulo. Téctum parcial. Infratéctum columelado, con columelas muy finas. Superficie reticulada, con lúmenes de menos de $0,5 \mu \mathrm{m}$ de diámetro y muros densamente equinulados (fig. 5: 11-14).

Referencias: se desconocen.

No se han encontrado referencias sobre la posible alergenicidad de esta especie. Tampoco ha sido citada como aerovagante.

\section{Ricinus communis L. (Subcosmopolita)}

Polen trizonocolporado. Isopolar, radiosimétrico. Circular en v.p. y c.o.e., de circular a ligeramente elíptico en v.e. y c.o.m. Oblado-esferoidal. Tamaño mediano. Aberturas compuestas; ectoaberturas de tipo colpo, terminales, bordeadas de un estrecho margo liso de aproximadamente $1 \mu \mathrm{m}$ de anchura; endoaberturas de tipo poro, lalongadas, elípticas, de $3(-4) \times 10-17 \mu \mathrm{m}$, rectangulares o elipsoidales. Membrana apertural difícil de apreciar. Exina de 2 $\mu \mathrm{m}$ de grosor, con la sexina más gruesa que la nexina, formando ésta última costillas a nivel de las endoaberturas. Téctum contínuo. Infratéctum columelado. Superficie perforada. Como elementos supratectales aparecen pequeños gránulos (fig. 6: 1-4).

Referencias: Pla Dalmau (1961), Lewis et al. (1983), Gálvez y Ubera (1985), Mateu et al. (1987), López y Díez (1987).

El género Ricinus es considerado como altamente alergógeno por Lewis et al. (1983), sin embargo Pla Dalmau (1961) consideró a esta especie con un grado de alergenicidad bajo. Citada como aerovagante por Cabezudo et al. (1994) en Málaga.

\section{ACERACEAE}

Acer negundo L. (este de Norteamérica)

Polen trizonocolpado. Isopolar, radiosimétrico. Circular lobulado en v.p. y c.o.e., sinuaperturado; elíptico en visión ecuatorial y corte óptico meridia- 

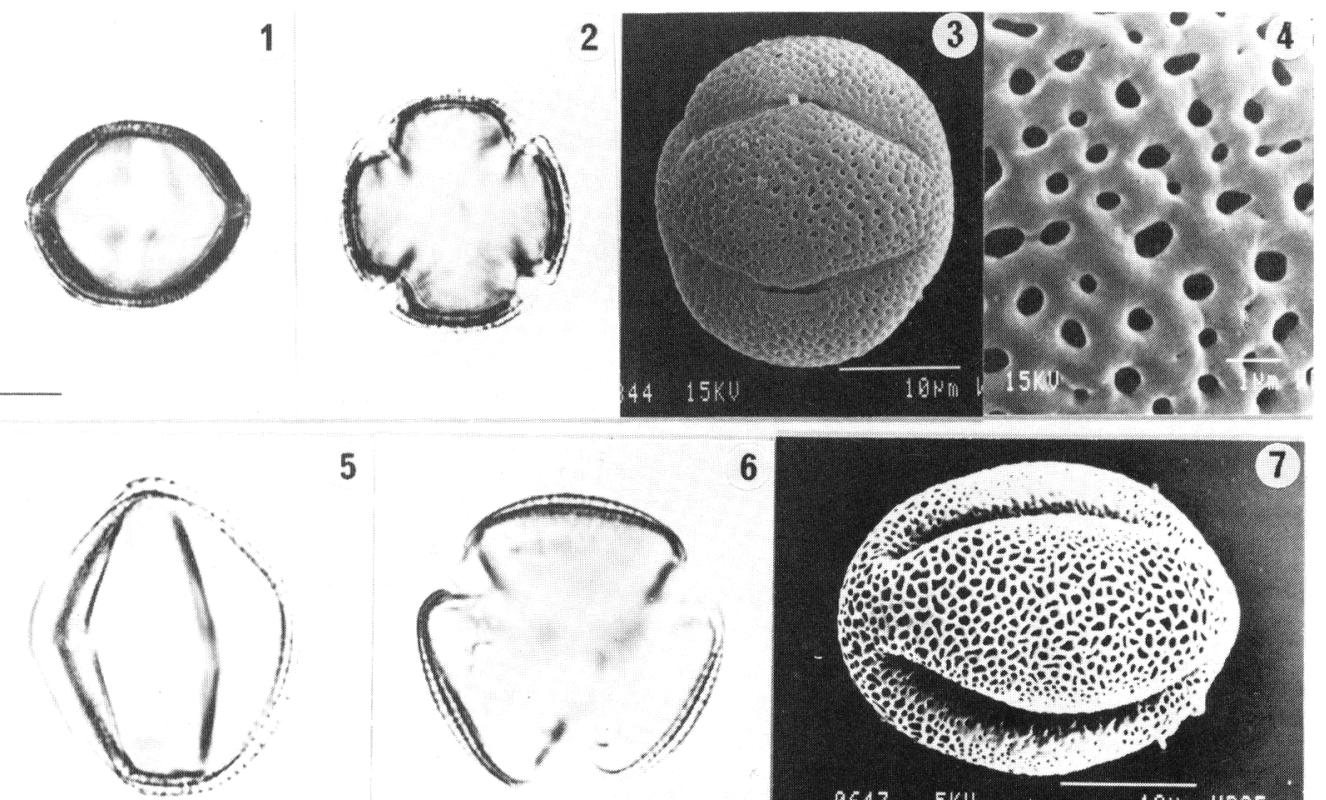

5

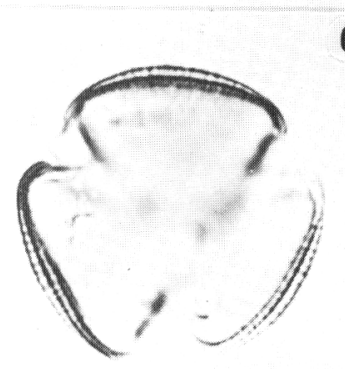

6
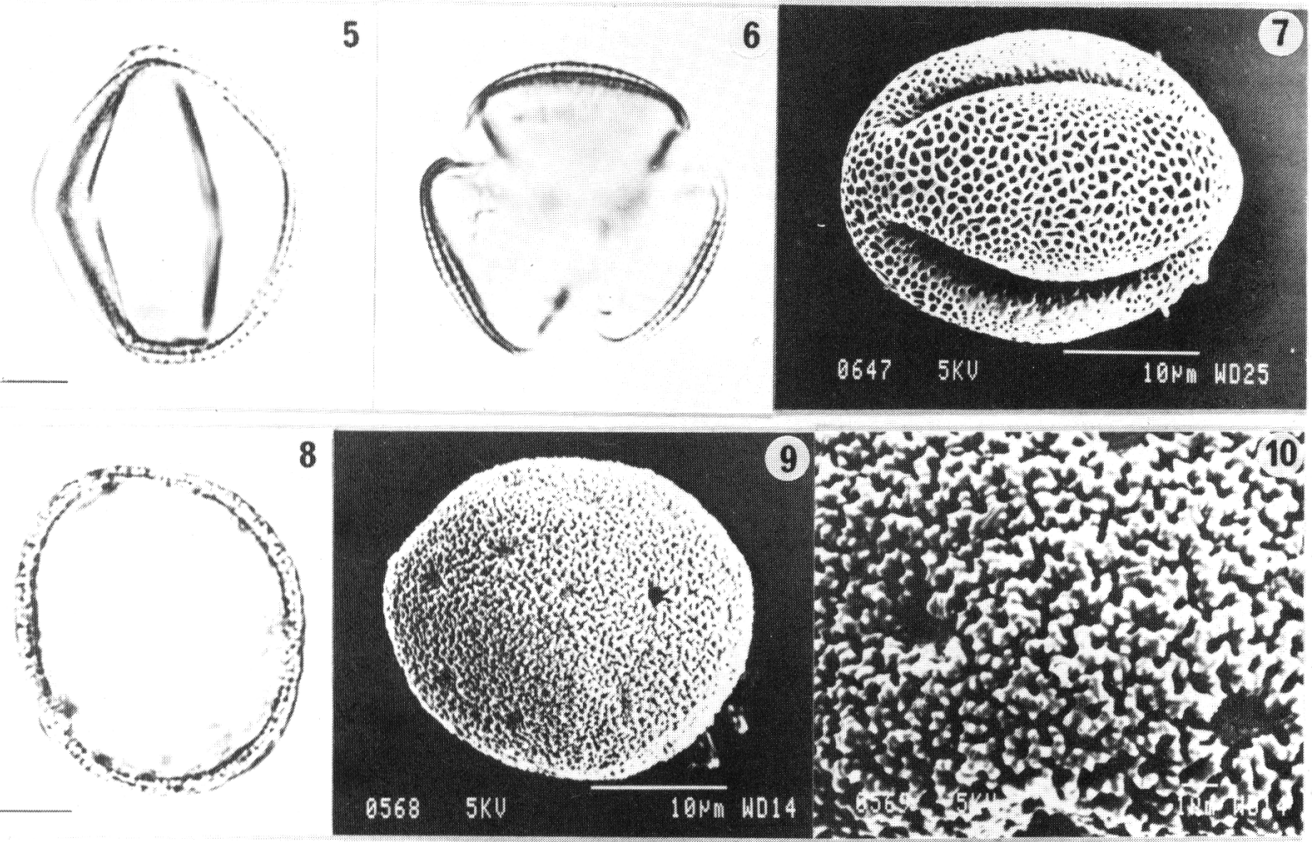

9) 5x B.?

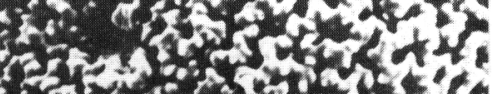

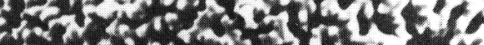
(c)

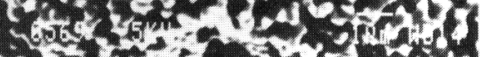
11
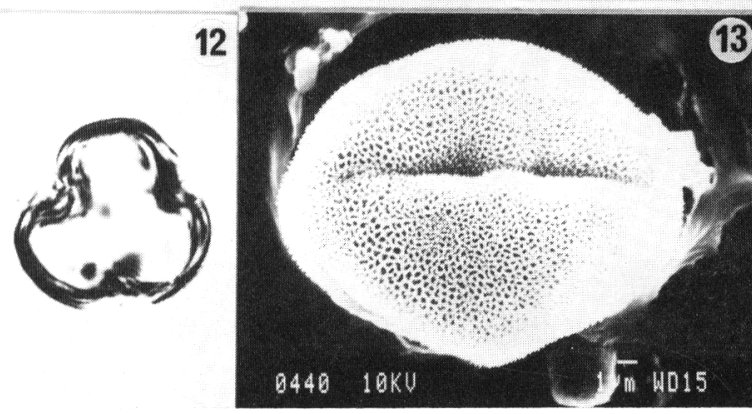

13 K (3) 60.04

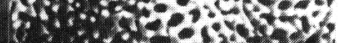
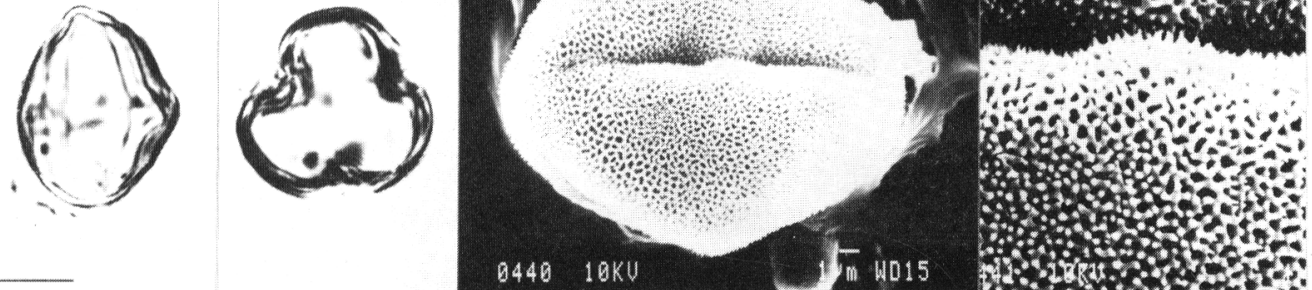

Figura 5. Ceratonia siliqua: 1) v.e. y c.o.m. 2) v.p. y c.o.e. 3) aspecto general del polen. 4) Detalle de la superficie. Gleditsia triacanthos: 5) v.e. y c.o.m. 6) v.p. y c.o.e. 7) Aspecto general del polen. Buxus balearica: 8) c.o. 9) Aspecto general del polen. 10) Detalle de la superficie y abertura. Acalypha willkesiana: 11) v.e. y c.o.m. 12) v.p. y c.o.e. 13) Aspecto general del polen. 14) Detalle de la ornamentación y abertura. Escala MO: $10 \mu \mathrm{m}$. 
no. De subprolado a oblado-esferoidal. Tamaño mediano. Aberturas simples, de tipo colpo, terminales. Membrana apertural difícil de apreciar. Exina de 1-1,5 $\mu \mathrm{m}$ de grosor, con la sexina más gruesa que la nexina. Téctum contínuo. Infratéctum columelado. Superficie rugulada, con los elementos más largos dispuestos en la zona ecuatorial (fig. 6: 5-8).

Referencias: Erdtman (1952), Pla Dalmau (1961), Biesboer (1976), Clarcke \& Jones (1980), Lewis et al. (1983), Roure (1985), Mateu et al. (1987), Díaz de la Guardia et al. (1988), Pino y Díez (1993).

Especie citada como alergógena por Izco et al. (1972), Sáenz (1978), Eriksson (1978), Lewis et ał. (1983), Halse (1984) y Rogers (1989). El género Acer es citado como aerovagante en numeroso trabajos sobre aerobiología.

\section{ANACARDIACEAE}

\section{Pistacia lentiscus L. (Región Mediterránea)}

Polen polipantoporado. Apolar, radiosimétrico. Circular en corte óptico. Esferoidal. Tamaño de pequeño a mediano. Aberturas simples, de tipo poro, en número de 4-8 por grano de polen, de contorno irregular y 1,5-3 $\mu \mathrm{m}$ de diámetro. Membrana apertural granulosa. Exina de 1-1,5 $\mu \mathrm{m}$ de grosor, con la sexina más gruesa que la nexina. Téctum parcial. Infratéctum columelado. Superficie irregularmente perforado-reticulada, con lúmenes de menos de $1 \mu \mathrm{m}$. Aparecen espínulas, densamente dispuestas, como elementos supratectales (fig. 6: 9-11).

Referencias: Erdtman (1952), Pla Dalmau (1961), Domínguez et al. (1984), Roure (1985), Díez (1987c).

La capacidad alergizante de esta especie fue puesta de manifiesto por Keyman et al. (1987). Igualmente ha sido mencionada como alergógena por Sáenz (1978), Domínguez et al. (1984) y Lewis et al. (1983). Citada como aerovagante por Domínguez et al. (1984), Belmonte i Soler (1988) y Torreguitart Zorrilla et al. (1990) y por Cabezudo et al. (1994) en Málaga.

\section{Schinus molle L. (Sudamérica)}

Polen trizonocolporado. Isopolar, radiosimétrico. Subtriangular en v.p. y c.o.e., angulaperturado; circular en v.e. y c.o.m. De oblado-esferoidal a prolado-esferoidal. Tamaño mediano. Aberturas compuestas; ectoaberturas de tipo colpo, terminales; endoaberturas de tipo poro, lalongadas, de 1-2 x 7-10 $\mu \mathrm{m}$. Membrana apertural granulosa. Exina de aproximadamente $2 \mu \mathrm{m}$ de grosor, con la sexina más gruesa que la nexina, si bien ésta adquiere mayor grosor a nivel de las endoaberturas para formar costillas. Téctum contínuo. Infratéctum columelado. Superficie estriada, con las liras psiladas y paralelamente dispuestas al eje polar (fig. 6: 12-15).

Referencias: Gálvez y Ubera (1985), Roure (1985), La-Serna Ramos et al. (1991), Pino y Díez (1993).

Especie citada como alergógena por Lewis $e t$ al. (1983) y Halse (1984).

\section{Spondias mombin L. (América tropical)}

Polen trizonocolporado. Isopolar, radiosimétrico. Circular lobulado en v.p. y c.o.e., sinuaperturado; elíptico en v.e. y c.o.m. De subprolado a proladoesferoidal. Tamaño de pequeño a mediano. Aberturas compuestas; ectoaberturas de tipo colpo, terminales; endoaberturas de tipo poro, lalongadas, de $1,5 \times 8-12 \mu \mathrm{m}$. Membrana apertural granulosa. Exina de 2-2,5 $\mu \mathrm{m}$ de grosor, con la sexina aproximadamente tan gruesa como la nexina, formando ésta última costillas a nivel de las endoaberturas. Téctum contínuo. Infratéctum columelado. Superficie estriada, con las liras psiladas y paralelamente dispuestas al eje polar (fig. $7: 1-4$ ).

Referencias: se desconocen.

No se han encontrado referencias sobre la posible alergenicidad de esta especie. Tampoco ha sido citada como aerovagante.

\section{SIMARUBACEAE}

Ailanthus altissima (Miller) Swingle (China)

Polen trizonocolporado. Isopolar, radiosimétrico. Triangular en v.p. y c.o.e., angulaperturado; elíptico obtuso en v.e. y c.o.m. De prolado a proladoesferoidal. Tamaño mediano. Aberturas compuestas; ectoaberturas de tipo colpo, subterminales, de 14-22 $\mu \mathrm{m}$ de longitud; endoaberturas de tipo poro, lalongadas, elípticas, de 1,5-3 x 4-6 $\mu \mathrm{m}$. Membrana apertural granulosa. Exina de 3-4 $\mu \mathrm{m}$ de grosor, con la sexina aproximadamente tan gruesa como la nexina, engrosándose ésta última a nivel de las endoaberturas para formar costillas. Téctum contínuo. Infratéctum columelado. Superficie estriado-reticulada, con las liras irregularmente 

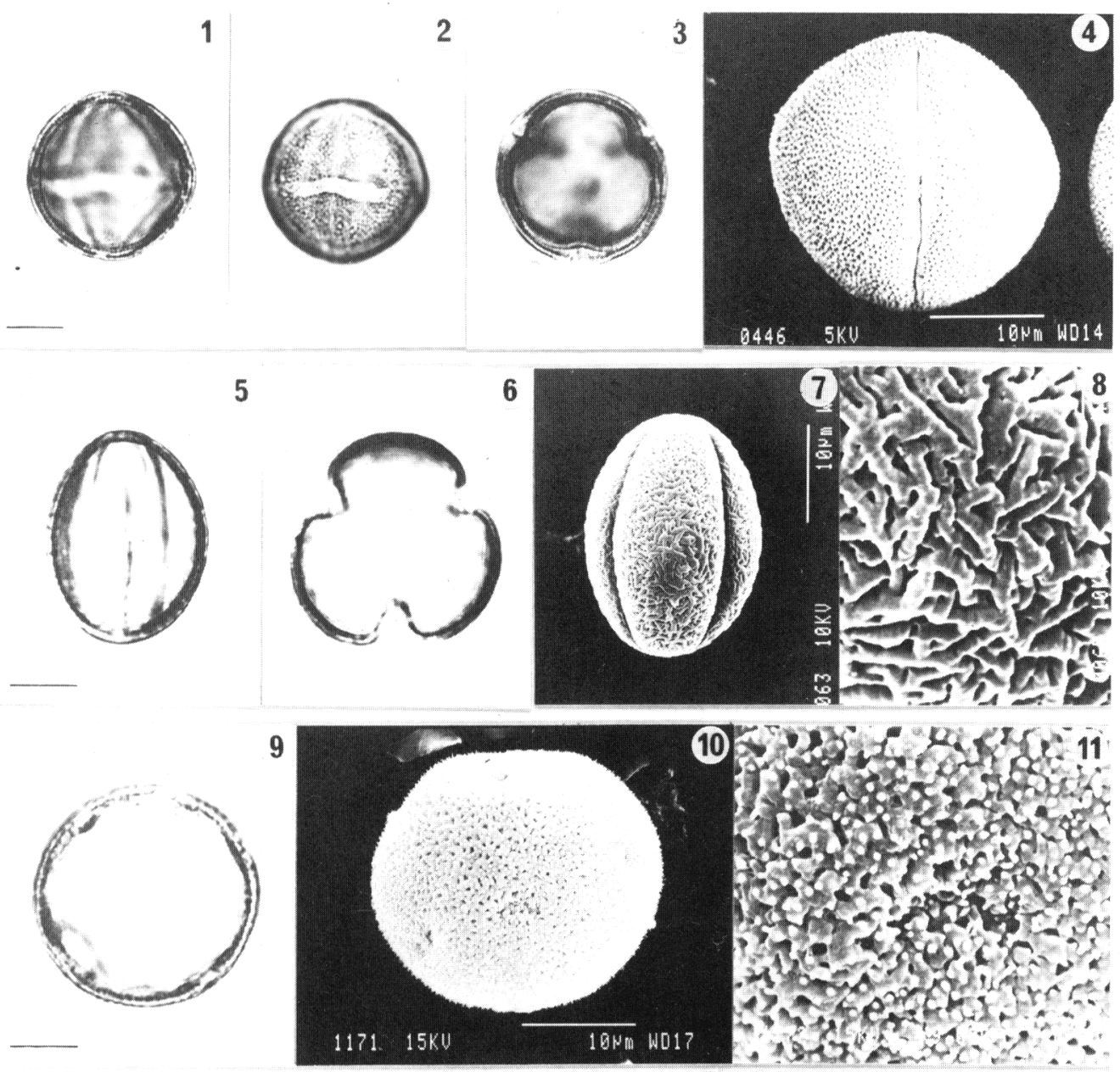

12

13

14

15
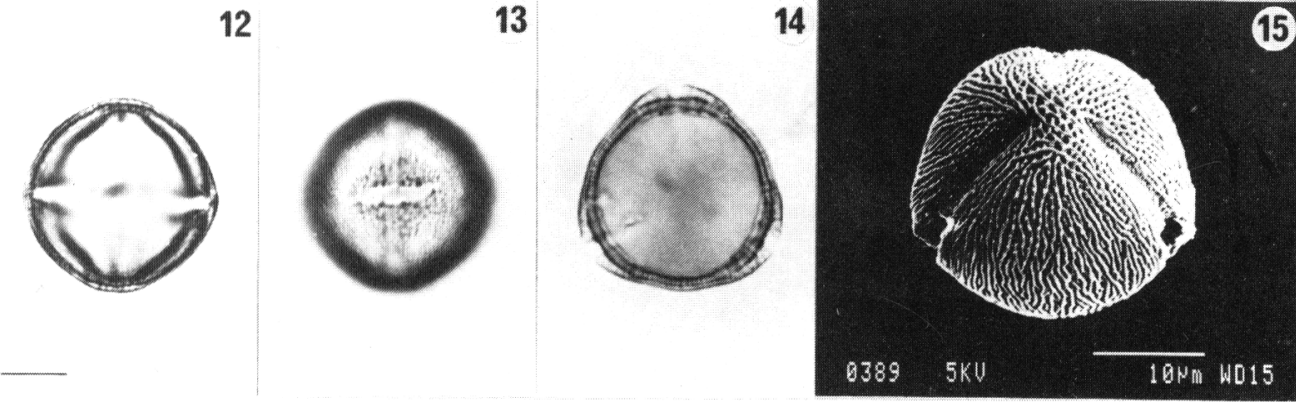

Figura 6. Ricinus communis: 1) v.e. y c.o.m. 2) Abertura. 3) v.p. y c.o.e. 4) Aspecto general del polen. Acer negundo: 5) v.e. y c.o.m. 6) v.p. y c.o.e. 7) Aspecto general del polen. 8) Detalle de la ornamentación. Pistacia lentiscus: 9) c.o. 10) Aspecto general del polen. 11) Detalle de la superficie y abertura. Schinus molle: 12) v.e. y c.o.m. 13) Abertura. 14) v.p. y c.o.e. 15) Aspecto general del polen. Escala MO: $10 \mu \mathrm{m}$. 
dispuestas (fig. 7: 5-8).

Referencias: Erdtman (1952), Lewis et al. (1983), Candau (1987c), Díaz de la Guardia et al. (1988).

Especie citada como alergógena por Muñoz Medina (1949), Lewis et al. (1983), Halse (1984) y Domínguez et al. (1984). También se encuentra citada como aerovagante por estos últimos autores.

\section{JUGLANDACEAE}

Carya pekan Engl. et Graebn. (sur de Norteamérica y México)

Polen trizonocolporado. Isopolar, radiosimétrico. Triangular en v.p. y c.o.e., angulaperturado; elíptico en v.e. y c.o.m. De oblado a suboblado, rara vez oblado-esferoidal. Tamaño de mediano a grande. Aberturas simples de tipo poro, de 3-5 $\mu \mathrm{m}$ de diámetro y contorno circular. Exina de 1,5-2,5 $\mu \mathrm{m}$ de grosor, siendo un poco más gruesa alrededor de los poros $($ c. $3 \mu \mathrm{m})$, con la sexina aproximadamente igual de gruesa que la nexina, engrosándose esta última alrededor de los poros para formar anillo. Téctum contínuo. Infratéctum sin columelas aparentes. Superficie densa y regularmente equinulada (fig. 7: 9-11). (1987).

Referencias: Lieux (1980), Mateu et al.

Especie citada como alergógena por Lewis et al. (1983), Halse (1984) y Rogers (1989); y como aerovagante por Durand \& Comtois (1989).

\section{Juglans regia L. (Himalaya y China)}

Polen polipantoporado. Isopolar o apolar, a veces heteropolar, radiosimétrico. De subcircular a subtriangular en v.p. y c.o.e., elíptico en v.e. y c.o.m. Oblado. Tamaño de mediano a grande. Aberturas simples, de tipo poro, circulares o ligeramente elípticas, de 3-5 $\mu \mathrm{m}$ de diámetro, en número de 316 por grano de polen, a menudo irregularmente distribuidas por la superficie del mismo. Exina de 2-3 $\mu \mathrm{m}$ de grosor, con la sexina igual o ligeramente más gruesa que la nexina, engrosándose ésta última a nivel de los poros para formar anillo. Téctum contínuo. Infratéctum con columelas poco patentes. Superficie densa y regularmente equinulada, con espínulas de muy pequeño tamaño (fig. 7: 1214).

Referencias: Pla Dalmau (1961), Domínguez et al. (1984), Díez (1987d).

Especie citada como alergógena por Pla
Dalmau (1961), Sáenz (1978), Lewis et al. (1983) y Domínguez et al. (1984). El género Juglans ha sido repetidamente citado como aerovagante.

\section{OLEACEAE}

Fraxinus pensylvanica Marshall (Norteamérica)

Polen tetrazonocolporado (colporoidado), a veces trizonocolporado (colporoidado). Isopolar, radiosimétrico. Cuadrado, a veces triangular, en v.p. y c.o.e., angulaperturado; elíptico en v.e. y c.o.m. De proladoa oblado-esferoidal. Tamaño pequeño. Aberturas compuestas; ectoaberturas de tipo colpo, terminales; endoaberturas de tipo poro, más o menos difusas. Membrana apertural granulosa. Exina de $1 \mu \mathrm{m}$ de grosor, con la sexina ligeramente más gruesa que la nexina. Téctum parcial. Infratéctum columelado. Superficie reticulada, con lúmenes de contorno irregular de hasta $1,5 \mu \mathrm{m}$ de longitud y los muros granulosos (fig. 7: 15-18).

Referencias: Lewis et al. (1983).

Especie citada como fuertemente alergógena por Lewis et al. (1983) y Halse (1984). El género Fraxinus ha sido ampliamente citado como alergógeno y aerovagante en numerosos trabajos de aerobiología (Macchia et al., 1991).

\section{Ligustrum lucidum Ait. (China, Corea y Japón)} Ligustrum ovalifolium Sieb. et Zucc. (Japón)

Polen trizonocolporado (colporoidado). Isopolar, radiosimétrico. Circular en v.p. y c.o.e., de subcircular a ligeramente elíptico en v.e. y c.o.m. De suboblado a prolado-esferoidal, rara vez subprolado. Tamaño mediano. Aberturas compuestas; ectoaberturas de tipo colpo, subterminales, de 10-14 $\mu \mathrm{m}$ de longitud en $L$. lucidum y de 17-20 $\mu \mathrm{m}$ en $L$. ovalifolium; endoaberturas difusas, de tipo poro. Membrana apertural escábrida en $L$. ovalifolium y granulosa en L. lucidum. Exina de 3$4 \mu \mathrm{m}$ de grosor, con la sexina unas 3 veces más gruesa que la nexina. Téctum parcial. Infratéctum columelado, con columelas gruesas y ensanchadas en el ápice. Superficie reticulada, con lúmenes de contorno irregular y de hasta $5 \mu \mathrm{m}$ de diámetro en L. lucidum y con lúmenes subcirculares de hasta 4 $\mu \mathrm{m}$ de diámetro en $L$. ovalifolium. En ambos casos los lúmenes del retículo dejan ver las columelas del infratéctum; muros psilados. En L. lucidum a veces se aprecian clavas en el interior de los lúmenes cuando se observan los granos de polen con el MEB (fig. 8: 1-4). 


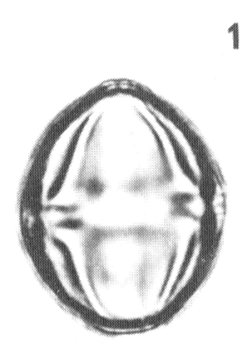

1

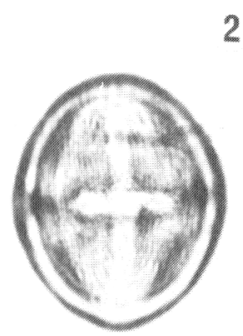

2

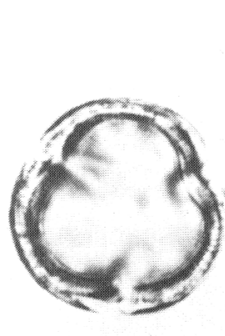

3

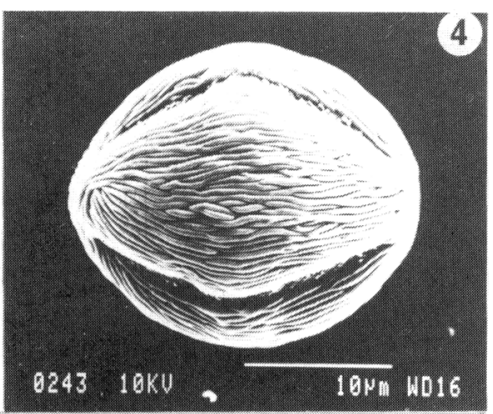

5
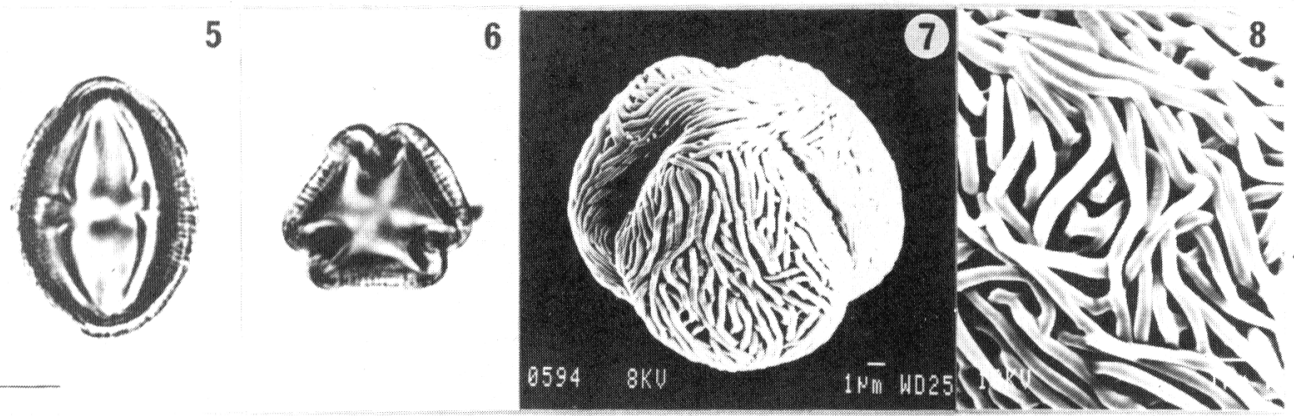

11

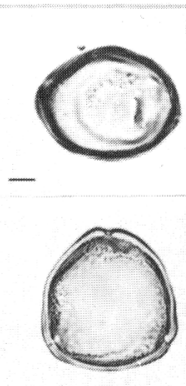

12
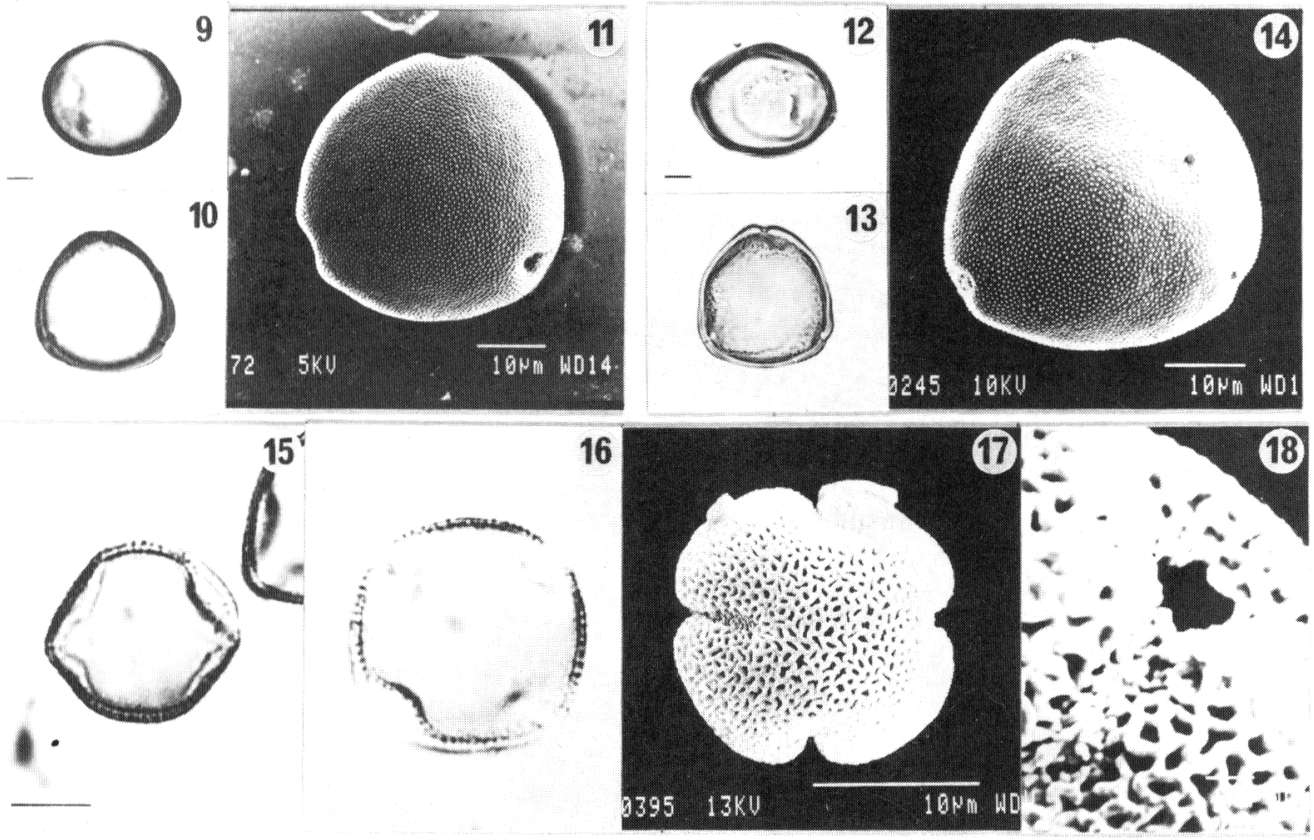

Figura 7. Spondias mombin: 1) v.e. y c.o.m. 2) Abertura 3) v.p. y c.o.e. 4) Aspecto general del polen. Ailanthus altissima: 5) v.e. y c.o.m. 6) v.p. y c.o.e. 7) Aspecto general del polen. 8) Detalle de la superficie. Carya pekan: 9) v.e. y c.o.m. 10) v.p. y c.o.e. 11) Aspecto general del polen. Juglans regia: 12) v.e. y c.o.m. 13) v.p. y c.o.e. 14) Aspecto general del polen. Fraxinus pensylvanica: 15) v. e. y c.o.m. 16) v.p. y c.o.e. 17) Aspecto general del polen 18) Detalle de la superficie y abertura. Escala MO: $10 \mu \mathrm{m}$. 
Referencias: L. lucidum : Domínguez et al. (1984). L. ovalifolium: Candau et al. (1981), Domíngez et al. (l.c.), La-Serna Ramos et al. (1991).

Especies citadas como alergógenas por Izco et al. (1972), Subiza (1980), Lewis et al. (1983), Halse (1984), Domínguez et al. (1984) y (Macchia et al., 1991). Y como aerovagantes por Pérez de Zabalza y Alvarez Calviño (1982) y Domínguez et al. (1984).

\section{Olea europaea L. (Región Mediterránea)}

Polen trizonocolporado (colporoidado). Isopolar, radiosimétrico. Subcircular en v.p. y c.o.e., de subcircular a ligeramente elíptico en v.e. y c.o.m. De suboblado a subprolado. Tamaño pequeño. Aberturas compuestas; ectoaberturas de tipo colpo, subterminales, de 6-10 $\mu \mathrm{m}$ de longitud; endoaberturas difusas, de tipo poro. Membrana apertural granulosa. Exina de 3-3,5 $\mu \mathrm{m}$ de grosor, con la sexina más gruesa que la nexina. Téctum parcial. Infratéctum columelado, con columelas gruesas. Superficie reticulada, con lúmenes de hasta $2,5 \mu \mathrm{m}$ de longitud y los muros estrechos y granulosos (fig. 8: 5-8).

Referencias: Pla Dalmau (1961), Domínguez et al. (1984), Candau (1987d), Nilsson (1988).

Especie citada como una de las causas más importantes de polinosis en la Región Mediterránea e incluida en la inmensa mayoría de los trabajos consultados tanto como alergógena como aerovagante (Spieksma, 1991). Citada por Cabezudo et al. (1994) como el tipo polínico que más incidencia tiene en la atmósfera de Málaga.

\section{CONCLUSIONES Y DISCUSIÓN}

De las 45 especies estudiadas, encontramos 11 de las que no tenemos referencias sobre trabajos anteriores, al menos en lo que a morfología polínica se refiere. En cuanto al resto de las especies, podríamos decir que, en general, no existen grandes discrepancias con la bibliografía consultada, si bien en algunos casos encontramos algunas diferencias que pasamos a comentar. Así, en Cocculus laurifolius observamos que las endoaberturas son lolongadas mientras que para Pino y Díez
(1990) aparecen lalongadas. Estos mismos autores describieron el polen de Platanus hispanica como angulaperturado en vista polar (v.p.) y corte óptico ecuatorial (c.o.e.), con los colpos terminales, mientras que en nuestras muestras nos aparece planaperturado y con colpos subterminales (fig. 1: 4-6). En el caso de Phytolacca dioica, los autores anteriormente citados describen la superficie del grano de polen escábrida y, sin embargo, hemos observado espínulas claramente diferenciadas como elementos supratectales (fig. 2: 14).

Pla Dalmau (1961) describió los granos de polen de Tamarix gallica como sincolpados, sin que en nuestras muestras hayamos encontrado este tipo polínico y, en el caso de Ceratonia siliqua observamos que la mayoría de los granos de polen eran tetrazonocolporados y no tricolpados como sugería este último autor. Por otra parte, no se han encontrado los tipos 2-sincolpado, espiraperturado ni pericolpado descritos por Ferguson (1980), aunque sí un tipo 4-loxocolporado que no había sido mencionado anteriormente.

Domínguez et al. (1984) y Roure (1985) describieron los granos de polen de Pistacia lentiscus como isopolares, sin embargo, al estar los poros regularmente repartidos por toda la superficie creemos más conveniente el considerarlos como apolares (fig. 6:10). También observamos que los elementos supratectales que presentan estos granos de polen son espínulas y no gránulos como los describe Díez (1987d). Igualmente, Roure (1985) considera los granos de polen de Schinus molle con simetría bilateral, reconociendo por otra parte, la existencia de 3 aberturas colporadas, carácter éste que le confiere simetría radial.

En el caso de Ailanthus altissima encontramos que los granos de polen presentan la superficie estriado-reticulada mientras que Díaz de la Guardia et al. (1988) la consideraron como reticulada (fig. 7: 7-8).

Por otra parte, Mateu et al. (1987) estudiaron los granos de polen de Carya pekan, 

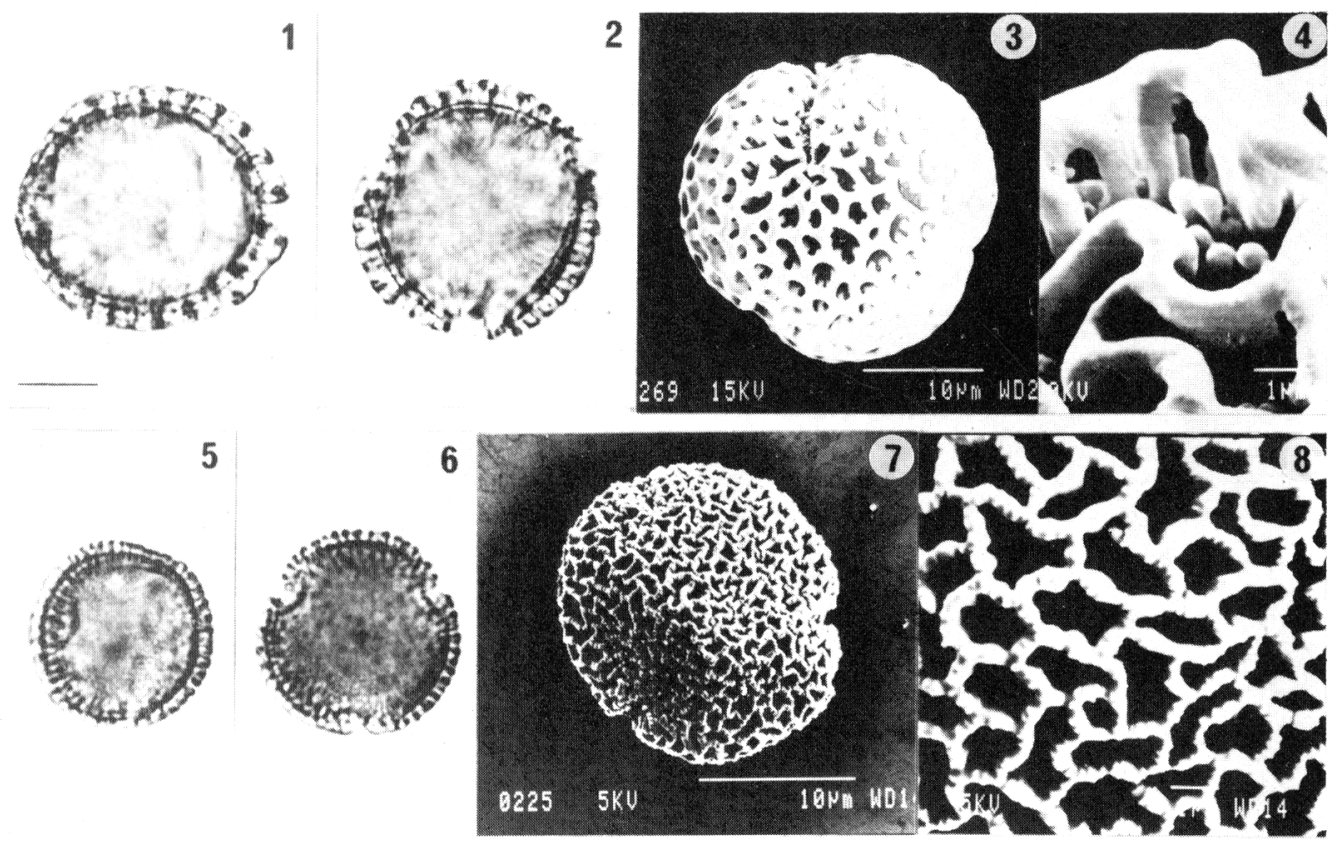

Figura 8. Ligustrum lucidum: 1) v.e. y c.o.m. 2) v.p. y c.o.e. 4) Detalle de la superficie. Ligustrum ovalifolium: 3) aspecto general del polen. Olea europaea: 5) v.e. y c.o.m. 6) v.p. y c.o.e.7) Aspecto general del polen. 8) Detalle de la superficie. Escala MO: $10 \mu \mathrm{m}$.

describiéndolos como circulares tanto en v.p. y c.o.e. como en v.e. y c.o.m., dato éste incomprensible si tenemos en cuenta que dan como valor medio de $\mathrm{P} / \mathrm{E}=0,70$, por lo que creemos que dicha apreciación se debe a un error.

Por último, cabe comentar que, aunque no encontramos referencias sobre la morfología polínica de Salix x chrysocoma y $S$. $x$ rubens, observamos que su aspecto es muy similar a la de las especies parentales estudiadas por Blanco (1988).

En otro orden de cosas, hemos podido comprobar que la inmensa mayoría de las especies que aquí se estudian han sido repetida- mente citadas como alergógenas en gran catidad de trabajos, aunque son escasos los estudios que incluyen pruebas sobre la capacidad alergizante de las mismas. En general se trata de especies cuyos pólenes son citados habitualmente como aerovagantes en numerosos trabajos de aerobiología y calendarios polínicos, aunque hay que tener en cuenta que es probable que los pólenes de especies exóticas pasen desapercibidos, engrosando el capítulo de los indeterminados o que, por su similar morfología, se incluyan dentro de grupos más amplios, como es el caso de Amaranthaceae o Chenopodiaceae. 


\begin{tabular}{|c|c|c|c|c|}
\hline & D & $\mathbf{P}$ & $\mathbf{E}$ & $\mathbf{P} / \mathbf{E}$ \\
\hline Acalypha willkesiana & - & $21,5-28(22,9 \pm 1,3)$ & $18,5-24(20,4 \pm 1,1)$ & $0,90-1,40(1,12 \pm 0,08)$ \\
\hline Acer negundo & - & $27-32(28,6 \pm 1,5)$ & $23-29(27,2 \pm 1,5)$ & $0,96-1,17(1,05 \pm 0,06)$ \\
\hline Ailanthus altissima & - & $20-27(25,3 \pm 1,9)$ & $13,5-23(17,9 \pm 2,1)$ & $1,08-1,73(1,38 \pm 0,16)$ \\
\hline Alternanthera bettzickiana & $14,5-18,5(16,1 \pm 1,4)$ & - & - & - \\
\hline Amaranthus caudatus & $18,5-26,5(22,5 \pm 1,7)$ & - & - & \\
\hline Antigonon leptopus & - & $22,5-25,5(23,9 \pm 0,8)$ & $17-21,5(18,8 \pm 1,3)$ & $1,03-1,42(1,26 \pm 0,09)$ \\
\hline Atriplex halimus & $16-22,5(18,7 \pm 1,4)$ & - & - & - \\
\hline Broussonetia papyrifera & $12-14,5(13,5 \pm 0,7)$ & - & - & - \\
\hline Buxus balearica & $29-36(32,7 \pm 2)$ & - & - & - \\
\hline Buxus sempervirens & $26-35(30,1 \pm 3)$ & - & - & - \\
\hline Carya pekan & - & $37,5-42,5(39,6 \pm 1,5)$ & $42,5-57,5(50,1 \pm 2,9)$ & $0,71-0,89(0,79 \pm 0,05)$ \\
\hline Casuarina cuninghamiana & - & $21-25,5(23,4 \pm 1,3)$ & $25-31(27,7 \pm 1,5)$ & $0,75-0,88(0,82 \pm 0,04)$ \\
\hline Celosia argentea & $23,5-30,5(27,8 \pm 1,7)$ & - & - & - \\
\hline Celtis australis & $31-43(37,6 \pm 3,4)$ & - & - & - \\
\hline Ceratonia siliqua & - & $21-27,5(23,9 \pm 1,8)$ & $20-27,5(23,2 \pm 1,9)$ & $0,84-1,36(1,03 \pm 0,13$ \\
\hline Cocculus laurifolius & - & $13,5-18,5(16,2 \pm 1)$ & $11-14,5(12,8 \pm 0,8)$ & $1,06-1,36(1,25 \pm 0,08)$ \\
\hline Fraxinus pensylvanica & - & $18,5-25(21,2 \pm 1,78)$ & $12-22,5(16,9 \pm 2,7)$ & $0,88-1,73(1,28 \pm 0,24)$ \\
\hline Gleditsia triacanthos & - & $25,5-34,5(30,9 \pm 2,4)$ & $14,5-27(19,8 \pm 2,6)$ & $1,11-1,95(1,56 \pm 0,23)$ \\
\hline Gomphrena globosa & $20-27(23,3 \pm 1,8)$ & - & - & - \\
\hline Juglans regia & - & $33-35(33,8 \pm 0,8)$ & $45,5-52(48,8 \pm 1,8)$ & $0,66-0,71(0,68 \pm 0,01)$ \\
\hline Kochia scoparia & $27-33(30,4 \pm 2,1)$ & - & - & - \\
\hline Ligustrum lucidum & - & $24-30(27,2 \pm 1,9)$ & $25-35(29,8 \pm 2,1)$ & $0,83-1,03(0,91 \pm 0,05)$ \\
\hline Ligustrum ovalifolium & & $25-36(29,2 \pm 3,3)$ & $25-34(29 \pm 2,1)$ & $0,83-1,17(1,01 \pm 0,11)$ \\
\hline Morus alba & $17-21 £ 19,7 \pm 0,9)$ & - & - & - \\
\hline Olea europaea & - & $17-24(26,6 \pm 1,8)$ & $14,5-25(19,3 \pm 2,2)$ & $0,82-1,33(1,08 \pm 0,13)$ \\
\hline Phytolacca dioica & - & $25,5-40(33,2 \pm 3,3)$ & $19-29(24,2 \pm 2,2)$ & $1,2-1,6(1,37 \pm 0,09)$ \\
\hline Pistacia lentiscus & $22,5-25,5(24,1 \pm 0,9)$ & - & - & - \\
\hline Platanus hispanica & - & $13,5-16(14,6 \pm 0,7)$ & $13-16(15,1 \pm 0,8)$ & $0,89-1,18(0,96 \pm 0,06)$ \\
\hline Populus alba & $24-32(27 \pm 2,3)$ & - & - & - \\
\hline Populus $\mathrm{x}$ canadensis & $17-31(25,6 \pm 2,9)$ & - & - & - \\
\hline Quercus ilex & - & $20,5-27(23,3 \pm 1,7)$ & $17-25(19,9 \pm 1,9)$ & $0,95-1,47(1,17 \pm 0,09)$ \\
\hline Quercus robur & - & $22-32(26,8 \pm 2,5)$ & $18-29(24 \pm 3,1)$ & $0,88-1,66(1,13 \pm 0,17)$ \\
\hline Quercus rotundifolia & - & $22-31,5(26,2 \pm 2,2)$ & $20-26,5(23,4 \pm 1,6)$ & $0,92-1,43(1,12 \pm 0,11)$ \\
\hline Ricinus communis & - & $26-30(28,5 \pm 1)$ & $27-31(29,1 \pm 0,9)$ & $0,92-1(0,97 \pm 0,02)$ \\
\hline Salix $\mathrm{x}$ chrysocoma & - & $17,5-29(22,3 \pm 2)$ & $13-19(16 \pm 2)$ & $1-1,89(1,42 \pm 0,26)$ \\
\hline Salix $x$ rubens & - & $27-34(29,9 \pm 1,4)$ & $23-28(25,5 \pm 1,3)$ & $1,07-1,47(1,21 \pm 0,09)$ \\
\hline Schinus molle & - & $25-32(28,1 \pm 1,6)$ & $25-31(28,3 \pm 1,6)$ & $0,92-1,03(0,99 \pm 0,02)$ \\
\hline Spondias mombim & - & $24-30,5(27,6 \pm 1,3)$ & $20,5-26,5(23,6 \pm 1,5)$ & $1,09-1,33(1,17 \pm 0,07)$ \\
\hline Tamarix africana & - & $16-23(20,7 \pm 1,7)$ & $12-20(16,7 \pm 2,1)$ & $0,95-1,55(1,25 \pm 0,16)$ \\
\hline Tamarix gallica & - & $16-24(21,1 \pm 1,8)$ & $13,5-18,5(16,6 \pm 1,4)$ & $0,95-1,55(1,27 \pm 0,15)$ \\
\hline Tamarix parviflora & - & $16-21,5(19,1 \pm 1,6)$ & $9,5-17,5(13,8 \pm 1,8)$ & $1,14-1,83(1,39 \pm 0,17)$ \\
\hline Tilia platyphyllos & - & $20-32(26,3 \pm 1,7)$ & $37-49(43,3 \pm 2,2)$ & $0,49-0,74(0,63 \pm 0,06)$ \\
\hline Tilia tomentosa & - & $19-22,5(20,6 \pm 1)$ & $36-46(41,1 \pm 2,3)$ & $0,43-0,55(0,49 \pm 0,02)$ \\
\hline Ulmus glabra & - & $23-27(25,8 \pm 1,2)$ & $30,5-40(34,6 \pm 2,4)$ & $0,58-0,78(0,69 \pm 0,03)$ \\
\hline Ulmus minor & - & $21,5-26,5(23,7 \pm 1,3)$ & $29,5-36(32,6 \pm 1,7)$ & $0,61-0,82(0,71 \pm 0,05)$ \\
\hline
\end{tabular}

Tabla 1. Valores máximos y mínimos de los ejes polar (P), ecuatorial (E), diámetro (D) y relación P/E de los taxones estudiados. Maximum and minimum values of polar $(P)$ and ecuatorial $(E)$ axis, diameter $(D)$ and $P / E$ relation of the studied taxa. 


\section{BIBLIOGRAFÍA}

AL-DOORY, Y., I.F. DOMSON, W.A. HOWARD \& R.M.SLY. -1980- Airborne fungi and pollens of the Washington D.C. metropolitan area. Annals of Allergy, 45: 360-367.

BELMONTE i SOLER, J. -1988- Espectre pollínic atmosfèric de Lleida. Actes del Simposi Internacional de Botánica Pius Font i Quer, vol. II. Fameroàmia: 97-100.

BENTHEM, F., G.C.S. CLARCKE \& W. PUNT. 1984- Fagaceae. En W. PUNT \& G.C.S. CLARCKE (eds.). The northwest European pollen flora 4: 87-110. Elsevier, Amsterdam.

BIESBOER, D.D. -1976- Pollen morphology of the Aceraceae. Grana, 15: 19-27.

BLANCO, P. -1988- Estudio palinológico del género Salix L. (Salicaceae) en España. En J. CIVIS LLOVERA y M.F. VALLE (eds.) Actas del VI Congreso de Palinología. A.P.L.E.: 2939, Salamanca.

BUCHOLTZ, G.A., A.E. HENSEL, R.F. LOCKEY, D. SERBOUSEK \& R.P. WUNDERLIN. -1987Australian pine (Casuarina equisetifolia) pollen as an aeroallergen. Annals of Allergy, 59:5256.

CABEZUDO, B., M.M. TRIGO, M. RECIO y F.J. TORO. - 1994- Contenido polínico de la atmósfera de Málaga: años 1992 y 1993. Acta Bot. Malacitana, 19: 137-144.

CANDAU, P. -1987a- Chenopodiaceae. En B. VALDÉS, M.J. DÍEZ e I. FERNÁNDEZ (eds.). Atlas polínico de Andalucía Occidental: 103105. Instituto de Desarrollo Regional de la Universidad de Sevilla y Excma. Diputación de Cádiz, Sevilla.

CANDAU,' P. - 1987b- Moraceae. En B. VALDÉS, M.J. DÍEZ e I. FERNÁNDEZ (eds.). Atlas polínico de Andalucía Occidental: 92. Instituto de Desarrollo Regional de la Universidad de Sevilla y Excma. Diputación de Cádiz, Sevilla. CANDAU, P. -1987c- Simaroubaceae. En B. VALDÉS, M.J. DÍEZ e I. FERNÁNDEZ (eds.). Atlas polínico de Andalucia Occidental: 231 232. Instituto de Desarrollo Regional de la Universidad de Sevilla y Excma. Diputación de Cádiz, Sevilla.

CANDAU, P. -1987d- Oleaceae. En B. VALDÉS, M. J. DÍEZ e I. FERNÁNDEZ (eds.). Atlas polínico de Andalucía Occidental: 294-296. Instituto de Desarrollo Regional de la Universidad de Sevilla y Excma. Diputación de Cádiz, Sevilla

CANDAU, P., J. CONDE y A. CHAPARRO - 1981Palinología de Oleaceae, incidencia de su polen en el aire de Sevilla. Clínica de la polinosis. Bot. Macaronesica, 8-9: 89-102.

CHRISTENSEN, P.B. \& BLACKMORE 1988- The Northwest European Pollen Flora, 40: Tiliaceae. Review of Palaeobotany and Palynology, 57: 33-43.

CLARCKE, G. C. S. \& M.R. JONES -1980Aceraceae. En W. PUNT \& G. C. S. CLARCKE (eds.). The northwest european pollen flora 2: 181-193. Elsevier, Amsterdam.

D'AMATO, G. A. \& F. TH. M. SPIEKSMA. -1991Allergenic Pollen in Europe. Grana, 30: 67-70. DÍAZ DE LA GUARDIA, C., G. BLANCA y R.M. NIETO -1988- Aportación al estudio palinológico de la flora ornamental de la ciudad de Granada (España). Acta Bot. Malacitana, 13: 354-362.

DÍEZ, M.J. -1987a- Ulmaceae. En B. VALDÉS, M. J. DÍEZ e I. FERNÁNDEZ (eds.). Atlas polínico de Andalucía Occidental: 118-123. Instituto de Desarrollo Regional de la Universidad de Sevilla y Excma. Diputación de Cádiz, Sevilla.

DÍEZ, M.J. - 1987b- Salicaceae. En B. VALDÉS, M. J. DÍEZ e I. FERNÁNDEZ (eds.). Atlas polínico de Andalucía Occidental: 149-152. Instituto de Desarrollo Regional de la Universidad de Sevilla y Excma. Diputación de Cádiz, Sevilla.

DÍEZ , M.J. -1987c- Anacardiaceae. En B. VALDÉS, M. J. DÍEZ e I. FERNÁNDEZ (eds.). Atlas polínico de Andalucía Occidental: 230231. Instituto de Desarrollo Regional de la Universidad de Sevilla y Excma. Diputación de Cádiz, Sevilla.

DÍEZ, M.J. -1987d- Juglandaceae. En B. VALDÉS, M. J. DÍEZ e I. FERNÁNDEZ (eds.). Atlas polínico de Andalucía Occidental: 98-99. Instituto de Desarrollo Regional de la Universidad de Sevilla y Excma. Diputación de Cádiz, Sevilla.

DÍEZ, M.J. y R. SUAREZ - 1987- Fagaceae. En B. VALDÉS, M. J. DÍEZ e I. FERNÁNDEZ (eds.). Atlas polínico de Andalucía Occidental: 94-96. 
Instituto de Desarrollo Regional de la Universidad de Sevilla y Excma. Diputación de Cádiz, Sevilla.

DOMÍNGUEZ, E., J.L. UBERA y C. GALÁN 1984- Polen alergógeno de Córdoba. Publicaciones del Monte de Piedad y Caja de Ahorros de Córdoba.

DURAND ,L. \& P. COMTOIS. -1989- A comparative study between the Cour and the Burkard samplers. En P. COMTOIS (ed.). Aerobiology. Health. Enviroment. A Symposium: 93-101, Université de Montéal.

ERDTMAN, G. - 1945-Pollen morphology and plant taxonomy. III. Morina L. with addition on morphological terminology. Svensk. Bot. Tidskr. 39: 187-191.

ERDTMAN, G. -1952-Pollen morphology and plant taxonomy. Angiosperms. (An introducction to palynology- Almqvist \& Wiksell, Stockholm.

ERDTMAN, G. -1960- The acetolysis method. Svensk. Bot. Tidskr., 54: 561-564.

ERDTMAN, G. -1969- Handbook of palynology. An introduction to the study of pollen grains and spores. Muksgaard, Copenhagen.

ERIKSSON, N.E. -1978- Allergy to pollen from different deciduous trees in Sweden. Allergy, 33: 299-309.

FAEGRI, K. \& J. IVERSEN -1975- Textbook of pollen analysis. Munksgaard, Copenhagen.

FERGUSON, I.K. -1980- The pollen morphology of Ceratonia (Leguminosae: Caesalpinioideae). Kew Bull., 35: 273-279.

FERGUSON, I.K. -1987- A preliminary survey of the pollen exine stratification in the Caesalpinioideae. In CH. H. STIRTON (ed.). Advances in Legume Systematics III: 355-385. Royal Botanic Gardens, Kew.

FERNÁNDEZ, I. -1987a- Tamaricaceae. En B. VALDES, M. J. DIEZ e I. FERNANDEZ (eds.). Atlas polínico de Andalucía Occidental: 145146. Instituto de Desarrollo Regional de la Universidad de Sevilla y Excma. Diputación de Cádiz, Sevilla.

FERNÁNDEZ, I.-1987b- Caesalpiniaceae. En B. VALDES, M. J. DIEZ e I. FERNANDEZ (eds.). Atlas polínico de Andalucía Occidental: 184185. Instituto de Desarrollo Regional de la Universidad de Sevilla y Excma. Diputación de Cádiz, Sevilla.

GÁLVEZ, C. y J.L. UBERA -1985- Nuevas aporta- ciones al catálogo aeropalinológico de Córdoba: plantas exóticas. Anales Asoc. Palinol. Lengua Esp. 2: 215-225.

GERVAIS, P. y B. MILLET -1978- Atlas práctico de ecología médica aplicado a la alergología. Schering Corporation. U.S.A.

HALSE, R.R. -1984- Nomenclature of allergenic plants. I and II. Annals of Allergy, 53: 291-307.

HARLEY, M.M. \& I.K. FERGUSON -1982- Pollen and taxonomy of the tribe Menispermae (Menispermaceae). Kew Bull, 37: 353-366.

HIDEUX, M. \& I.K. FERGUSON - 1975 Stéréostructure de l'exine des saxifragales: proposition d'une description logique et schemátique. Bull. Soc. Bot. France, 122: 5767.

ICKOVIC, M.R. \& M. THIBAUDON -1991Allergenic significance of Fagaceae pollen. En G. D'AMATO, F. Th. M. SPIEKSMA \& S. BONNINI (eds.). Allergenic pollen and pollinosis in Europe: 98-108. Blackwell Scientific Publications.

IZCO, J., M. LADERO y C. SAENZ -1972- Flora alergógena de España. Distribución, descripción e interés medico alergológico de las especies resposables de síndromes alérgicos. Anales de la Real Academia de Farmacia 38: 521570.

KEYMAN, N., C. GELLER-BERNSTEIN, Y. WAISEL, A. BEJERANO, A. SHOMER-ILAN \& R. TAMIR -1987-Positive skin test to pollen extracts of four species of Pistacia in Israel Clinical Allergy 17: 243-249.

KING, T.P. \& P.S. NORMAN -1986- Standarized extracts weeds. Clin. Rev. Allergy, 4: 425-433.

LA-SERNA RAMOS, I., M.D. DOMÍNGUEZ SANTA, B. MENDEZ PÉREZ, J.R. ACEBES GINOVES y P.L. PÉREZ DE PAZ. - 1991- Contribución al atlas palinológico de la comarca Santa Cruz-La Laguna (Tenerife: Islas Canarias). Boletim da Sociedade Broteriana, 64: 99 134.

LEWIS, W.H., P. VINAY \& V.E. ZENGER -1983Airborne and allergenic pollen of North América. The Johns Hopkins University Press, Baltimore.

LIEUX, M.H.-1980- An atlas of trees, shrubs and woody vines of Louisiana and other southeastern states. Part II. Platanaceae to Betulaceae. Pollen et Spores, 22: 191-241. 
LÓPEZ, C. y M.J. DÍEZ -1987- Euphorbiaceae. En B. VALDES, M. J. DIEZ e I. FERNANDEZ (eds.). Atlas polínico de Andalucía Occidental: 212-218. Instituto de Desarrollo Regional de la Universidad de Sevilla y Excma. Diputación de Cádiz, Sevilla.

MACCHIA, L., M.F. CAIAFFA, G. D’AMATO \& A. TURSI. -1991- Allergenic Significance of Oleaceae Pollen. En G. D'AMATO, F. Th. M. SPIEKSMA \& S. BONNINI (eds.). Allergenic pollen ad pollinosis in Europe: 87-93. Blackwell Scientific Publications.

MATEU, I., A. AGUILELLA y J.M. AZCARRAGA -1987- Pólenes del Jardín Botánico de Valencia. I. Anales Asoc. Palinol. Lengua. Esp., 3: 73-90.

MATTHIESEN, F., H. IPSEN \& H. LØWENSTEIN. -1991- Pollen Allergens. En G. D’AMATO, F. Th. M. SPIEKSMA \& S. BONNINI (eds.). Allergenic pollen ad pollinosis in Europe:, 3644. Blackwell Scientific Publications.

MELVILLE, R. -1981- Surface tension, diffusion and the evolution and morphogenesis of pollen aperture patterns. Pollen et Spores, 23: 179203.

MICHEL, F.B., H. DHIVERT, J.P. MARTY, M.C. ALQUIE, P. COUR \& B. GUERIN -1978Pollinoses hivernales. Rev. Franc. Allergol., 18: 83-88.

MUÑOZ MEDINA, J. -1949- Una introducción al estudio de los alergenos polínicos de Granada. Anales de la Real Academia de Medicina de Granada, 13: 15-27.

NILSSON , S. -1988- A survey of the pollen morphology of Olea with particular reference to Olea europaea sens. lat. Kew Bull., 43: 303315 .

O'ROURKE, M.K. -1989- Relationships between airborne pollen concentrations and weather parameters in an arid environment. En 'P. COMTOIS (ed.). Aerobiology. Health. Enviroment. A Symposium: 55-76, Université de Montéal.

PARDO, C. -1990- Tipos harmomegatas en Gleditschia triacanthos. (Papilionaceae, Caesalpinioideae). En G. BLANCA, C. DÍAZ DE LA GUARDIA, M.C. FERNÁNDEZ, M. GARRIDO, M.I. RODRÍGUEZ-GARCÍA y A.T. ROMERO (eds.). Polen, esporas y sus aplicaciones:, 211-216.
PÉREZ DE ZABALZA, A.I. y A. ÁLVAREZ CALVIÑO. -1982- Estudio anual del polen atmósferico de la ciudad de Pamplona. En N. SOLE DE PORTA y M. SUAREZ CERVERA (eds.). Actas del IV Simposio de Palinología. A.P.L.E.: 233-249.

PINO, J.A. y M.J. DÍEZ -1990- Aportación a la palinología de plantas ornamentales de Sevilla. I. Lagascalia, 15: 199-221.

PINO, J.A. y M.J. DÍEZ -1993- Aportación a la palinología de plantas ornamentales de Sevilla. III. Lagascalia, 17(1): 87-103.

PLA DALMAU, J.M. -1961- Polen. Talleres gráficos D. C. P., Gerona.

PUNT, W. \& M. MALOTAUX - 1984- Cannabaceae, Moraceae and Urticaceae. En W. PUNT \& G. C. S. CLARCKE (eds.). The northwest european pollen flora, 4: 23-44. Elsevier, Amsterdam.

PUNT, W., S. BLACKMORE, S. NILSSON \& A. LE THOMAS. -1994- Glossary of pollen and spore terminology. LPP contributions series, 1. Utrecht.

REITSMA, T. -1969- Size modification of recent pollen grains under different treatments. Rev. Palaeobot. Palynol., 9: 175-202.

REITSMA, T. -1970- Suggestions towards unification of descriptive terminology of Angiosperms pollen grains. Rev. Palaeobot. Palynol., 10: 39-60.

ROGERS, C.A. -1989- Seasonal patterns of allergenic pollen at Toronto. En P. COMTOIS (ed.). Aerobiology. Health. Enviroment. A Symposium: 77-91, Université de Montéal.

ROURE, J.M. -1985- Palinología Ibérica. Fam. 1 a 20. Cupressaceae a Betulaceae. Orsis, 1: 43-69.

SAA, M.P., M.J. AIRA y F. DIAZ-FIERROS -1985Aportación al estudio palinológico del género Quercus en Galicia. Anales Asoc. Palinol. Lengua Esp., 2: 289-300.

SAENZ, C. -1973- Estudios palinológicos sobre Quercus de la España Mediterránea. Bol. Soc. Esp. Hist. Nat., 71: 315-329.

SAENZ, C. -1978- Polen y esporas. Editorial Blume, Madrid.

SAENZ, C. -1980- Polen de la flora de Doñana (Huelva, España). Lazaroa, 2: 191-270.

SINGH, A.B. \& C.R. BABU -1981-Variations in the atmospheric pollen spectra of Dhelhi region, India. Grana, 20: 191-195.

SPIEKSMA, F. Th. M. -1991- Regional European 
Pollen Calendars. En G. D' AMATO, F. Th. M. SPIEKSMA \& S. BONNINI (eds.). Allergenic pollen ad pollinosis in Europe: 49-65. Blackwell Scientific Publications.

STANLEY, J. \& C. LINSKEN -1974- Pollen. Biology biochemistry and management. Springer-Verlar, Berlin.

STEBBINS, G.L. -1974- Flowering plants. Evolution above the species level. Harvard University Press.

STOCKMARR, J. -1974- SEM studies on pollen grains of north european Ulmus species. Grana, 14: 103-107.

SUBIZA, E. -1980- Aerobiología: Los pólenes. Departamento de Alergia Abelló.

SURINYACH, R., P. MONSERRAT y R. FONT 1955- Epidemiología de la polinosis en Barcelona. Anales, Secc. Medicina, 42: 36-62.

TORREGUITART ZORRILLA, A., J. BELMONTE SOLER y J.M. ROURE NOLLA. -1990- Estudio comparativo del contenido polínico atmosférico en las Islas Baleares. En G. BLANCA, C. DIAZ DE LA GUARDIA, M. C. FERNÁNDEZ, M. GARRIDO, M. I. RODRÍGUEZ-GARCÍA y A. T. ROMERO (eds.). Polen, esporas y sus aplicaciones: $377-384$.

TRIGO, M.M. -1989- Aportación al estudio palinológico de la flora ornamental de la ciudad de Málaga: Gimnospermas. Acta Bot. Malacitana, 14: 238-244.

TRIGO, M.M., M. RECIO y B. CABEZUDO. -
1992- Sobre la palinología de algunas especies endémicas e interesantes de Andalucía Oriental. V. Acta Bot. Malacitana, 17: 267-277.

VAN CAMPO, M. -1957- Palinologie africaine I. Bull. Inst. Franç. Afrique Noire, sér. A, Sci. Nat., 20: 659-678.

VAN CAMPO, M. -1958- Palinologie africaine II. Bull. Inst. Franç. Afrique Noire, sér. A, Sci. Nat., 20: 753-760.

WODEHOUSE, R.P. -1935- Pollen grains. McGraw-Hill, New York.

ZAVADA, M. -1983- Pollen morphology of Ulmaceae. Grana, 22: 23-30.

Aceptado para su publicación en Mayo de 1994

Dirección de las autoras. M.M. Trigo: Dpto. Biología Vegetal. Facultad de Ciencias. Universidad de Málaga. Apdo. 59. 29080, Málaga. Fax, (95) 213 19 44. I. Fernández: Dpto. de Botánica. Facultad de Ciencias. Avda. Reina Mercedes, s/n. 41012, Sevilla. 